\title{
Quasi-Exactly Solvable Schrödinger Operators in Three Dimensions
}

\author{
Mélisande FORTIN BOISVERT
}

Department of Mathematics and Statistics, McGill University, Montréal, Canada, H3A 2K6

E-mail: boisvert@math.mcgill.ca

URL: http://www.math.mcgill.ca/boisvert/

Received October 01, 2007, in final form November 02, 2007; Published online November 21, 2007

Original article is available at http://www.emis.de/journals/SIGMA/2007/109/

\begin{abstract}
The main contribution of our paper is to give a partial classification of the quasiexactly solvable Lie algebras of first order differential operators in three variables, and to show how this can be applied to the construction of new quasi-exactly solvable Schrödinger operators in three dimensions.
\end{abstract}

Key words: quasi-exact solvability; Schrödinger operators; Lie algebras of first order differential operators; three dimensional manifolds

2000 Mathematics Subject Classification: 81Q70; 22E70; 53C80

\section{Introduction}

Recall that a Schrödinger operator on a $n$-dimensional Riemannian manifold $(\mathbf{M}, g)$ is a second order linear differential operator of the form

$$
\mathcal{H}_{0}=-\frac{1}{2} \Delta+U
$$

where $\Delta$ is the Laplace-Beltrami operator and $U$ is the potential function for the physical system under consideration. A question of fundamental interest in quantum mechanics is to construct eigenfunctions $\psi$ of the Schrödinger operator $\mathcal{H}_{0}$. One approach to this problem, which is based on the representation theory of Lie algebras, is to consider Schrödinger operators $\mathcal{H}_{0}$ which are quasi-exactly solvable, in a sense that will be defined below.

We begin by considering the case of a general linear second-order differential operator $\mathcal{H}$, given in local coordinates by

$$
\mathcal{H}=\sum_{i, j=1}^{n} A^{i j} \partial_{i} \partial_{j}+\sum_{i=1}^{n} B^{i} \partial_{i}+C .
$$

The operator $\mathcal{H}$ is said to be Lie algebraic if it is an element of the universal enveloping algebra of $\mathfrak{g}$, a finite dimensional Lie algebra of first order differential operators. More explicitly,

$$
\mathcal{H}=\sum_{a, b=1}^{m} C_{a b} T^{a} T^{b}+\sum_{a=1}^{m} C_{a} T^{a}+C_{0},
$$

where

$$
T^{a}=v^{a}+\eta^{a}, \quad 1 \leq a \leq m,
$$

is a basis or $\mathfrak{g}$. In (2), the operators $v^{1}, \ldots, v^{m}$ are vector fields, and $\eta_{1}, \ldots, \eta_{m}$ are multiplication operators. A Lie algebra $\mathfrak{g}$ of first order differential operators is said to be quasi-exactly solvable 
if one can find explicitly a finite dimensional $\mathfrak{g}$-module $\mathcal{N}$ of smooth functions, i.e., if $\mathcal{N}=$ $\left\{h^{1}, \ldots, h^{r}\right\}$ with $T^{a}(\mathcal{N}) \subset \mathcal{N}$ for all $1 \leq a \leq m$. A Lie algebraic operator $\mathcal{H}$, is said to be quasi-exactly solvable if it lies in the universal enveloping algebra of a quasi-exactly solvable Lie algebra of first order differential operators. Obviously, $\mathcal{H}(\mathcal{N}) \subset \mathcal{N}$, i.e. the module $\mathcal{N}$ will be fixed by the operator $\mathcal{H}$. Moreover, if the functions contained in the module $\mathcal{N}$ are square integrable with respect to the Riemannian measure $\sqrt{g} d x^{1} \cdots d x^{n}$, where $g$ is the determinant of the covariant metric, the operator $\mathcal{H}$ is said to be a normalizable quasi-exactly solvable operator.

We can see from the above definitions that the formal eigenvalue problem for quasi-exactly solvable Schrödinger operators can be solved partially by elementary linear algebraic methods. Indeed, the operator $\mathcal{H}$ is self-adjoint with respect to the inner product associated to the standard measure, therefore the restriction of $\mathcal{H}$ to the finite dimensional module $\mathcal{N}$ is a Hermitian finite dimensional linear operator. Thus, one can in principle, compute $r=\operatorname{dim}(\mathcal{N})$ eigenvalues of $\mathcal{H}$, counting multiplicities, by diagonalizing the $r \times r$ matrix representing $\mathcal{H}$ in a basis of $\mathcal{N}$.

It seems that the concept of a "spectrum generating algebra" was first introduced by Goshen and Lipkin in [15] in 1959. However, this paper did not seem to have been be noticed by the community and, ten years later, spectrum generating algebras were independently rediscovered by two groups of physicists, see [2] and [5]. Their work was an impetus for further research in this area as one can see by browsing in the two volume set of reprints [3] and the conference proceedings [16]. A survey of the history and the contribution papers related to the spectrum generating algebras is given in the review paper of Böhm and Ne'eman, which appears at the beginning of [4]. In the early 1980's, Iachello, Levine, Alhassid, Gürsey and collaborators exhibited applications of spectrum generating algebras to molecular spectroscopy; a survey of the theory and applications is given in the book [17]. In these applications, both nuclear and spectroscopic, the relevant Hamiltonian is a Lie algebraic operator in the sense described previously. Finally, the analysis of a new class of Schrödinger operators, the quasi-exactly solvable class, was initiated in late 1980's by Shifman, Turbiner and Ushveridze, see [23, 24, 25]. A survey of the theory and applications of quasi-exactly solvable systems in physics is given in [26].

There exists a complete classification of quasi-exactly solvable Schrödinger operators in one dimension. In two dimensions, this classification is in principle complete. Indeed, all the Lie algebraic linear differential operators for which the formal spectral problem is solvable are known. The question of determining if the operator is equivalent to a Schrödinger operator will be discussed below. The main contribution of our paper is to extend these results to three dimensions by giving a partial classification of the quasi-exactly solvable Lie algebras of first order differential operator in three variables, and showing how this can be applied to the construction of new quasi-exactly solvable Schrödinger operators in three dimensions. Our work is based on the classification of finite dimensional Lie algebras of vector fields in three dimensions begun by Lie in [18] and completed by Amaldi in [1].

Let $g^{(i j)}$ be the contravariant metric of the manifold $\mathbf{M}$ in a local coordinate chart, $\widetilde{g}$ its determinant and $g$ the determinant of the covariant metric. In that setting, a Schrödinger operator reads locally as

$$
\mathcal{H}_{0}=-\frac{1}{2} \sum_{i, j=1}^{n}\left[g^{i j} \partial_{i j}+\partial_{i}\left(g^{i j}\right) \partial_{j}-\frac{g^{i j} \partial_{i}(\widetilde{g})}{2 \widetilde{g}} \partial_{j}\right]+U
$$

Recall that a quasi-exactly solvable second order operator is not, in general, a Schrödinger operator. However this operator might be equivalent to a Schrödinger operator in a way that preserves the formal spectral properties of the operators under consideration. The appropriate notion of equivalence, which will be used throughout our work, is the following. Two differential operators are locally equivalent if there is a gauge transformation $\mathcal{H} \rightarrow \mu \mathcal{H} \mu^{-1}$, with gauge factor $\mu=e^{\lambda}$, and a change of variables relating one to the other. In principle, it is possible to 
verify if a general second order differential operator $\mathcal{H}$ is equivalent to a Schrödinger operator with respect to this notion of equivalence. Indeed, every second order linear differential operator can be given locally by

$$
\mathcal{H}=-\frac{1}{2} \sum_{i, j=1}^{n} g^{i j} \partial_{i j}+\sum_{i}^{n} h^{i} \partial_{i}+U
$$

If the contravariant tensor $g^{(i j)}$ is non-degenerate, that is if $g$ does not vanish, the operator can be expressed as

$$
\mathcal{H}=-\frac{1}{2} \Delta+\vec{V}+U
$$

where $\vec{V}=b^{i} \partial_{i}$ is a vector field. For this operator to be locally equivalent to a Schrödinger operator, the vector field $\vec{V}$ has to be a gradient vector field with respect to the metric $g^{(i j)}$. Locally, this will be the case if and only if $\omega=g_{i j} b^{j} d x^{i}$, the one form associated to $\vec{V}$, is closed. For this reason, this condition is named the closure condition. Note that if $\vec{V}=\nabla(\lambda)$, the gauge factor is given by $e^{\frac{\lambda}{2}}$.

Given an operator of the form (1), the closure conditions can be easily verified provided the contravariant metric $g^{(i j)}$ is non-degenerate. Indeed, these conditions can be written as algebraic constraints on the coefficients $C_{a b}$ and $C_{c}$, and are the Frobenius compatibility conditions for an overdetermined system that will be described later.

An important point to keep in mind is that the class of quasi-exactly solvable operators is invariant under local equivalence. Indeed, suppose $\mathcal{H}$ is a quasi-exactly solvable operator which is gauge equivalent to an other operator $\mathcal{H}_{0}$ under the rescaling $\mu$. If $\mathcal{H}$ lies in the universal enveloping algebra of $\mathfrak{g}$, whose $\mathfrak{g}$-module is $\mathcal{N}$, one can easily show that $\mathcal{H}_{0}$ is quasi-exactly solvable with respect to the finite dimensional Lie algebra

$$
\widetilde{\mathfrak{g}}=\mu \cdot \mathfrak{g} \cdot \mu^{-1}=\left\{\mu \cdot T \cdot \mu^{-1} \mid T \in \mathfrak{g}\right\}
$$

which is isomorphic to $\mathfrak{g}$ and posses the finite-dimensional $\widetilde{\mathfrak{g}}$-module

$$
\widetilde{\mathcal{N}}=\mu \cdot \mathcal{N}=\{\mu \cdot h \mid h \in \mathcal{N}\} .
$$

Note however that the gauge factor is not necessarily unitary. Thus, a gauge transformation does not necessarily preserve the normalizability property of the functions in $\mathcal{N}$. Therefore, the class of normalizable quasi-exactly solvable operators is not invariant under our local equivalence. We now give an example of a normalizable quasi-exactly solvable Schrödinger operator in three variables.

Example 1. In this example we consider the quasi-exactly solvable Lie algebra $\mathfrak{g} \cong \mathfrak{s l}(2) \times \mathfrak{s l}(2) \times$ $\mathfrak{s l}(2)$. With the standard notation $p=\frac{\partial}{\partial x}, q=\frac{\partial}{\partial y}$ and $r=\frac{\partial}{\partial z}$, this Lie algebra representation can be spanned by the following first order differential operators

$$
\begin{aligned}
& T^{1}=p, \quad T^{2}=x p, \quad T^{3}=x^{2} p-x, \quad T^{4}=q, \quad T^{5}=y q, \\
& T^{6}=y^{2} q-y, \quad T^{7}=r, \quad T^{8}=z r, \quad T^{9}=z^{2} r-z .
\end{aligned}
$$

Then, the finite dimensional module of smooth functions

$$
\mathcal{N}_{m_{x} m_{y} m_{z}}:=\left\{x^{i} y^{j} z^{k} \mid 0 \leq i \leq m_{x}, 0 \leq j \leq m_{y}, 0 \leq k \leq m_{z}\right\}
$$

is a $\mathfrak{g}$-module provided $m_{x}=m_{y}=m_{z}=1$. With the following choice of coefficients, one constructs the quasi-exactly solvable operator

$$
\begin{aligned}
-2 \mathcal{H}= & \left(T^{1}\right)^{2}+\left(T^{2}\right)^{2}+2\left[\left(T^{3}\right)^{2}+\left(T^{4}\right)^{2}+2\left(T^{5}\right)^{2}+2\left(T^{6}\right)^{2}\right]+\left(T^{7}\right)^{2}+\left(T^{8}\right)^{2} \\
& +2\left(T^{9}\right)^{2}+\left\{T^{1}, T^{3}\right\}+\left\{T^{7}, T^{9}\right\}-2 T^{4}-2 T^{5}-4 T^{6}-8,
\end{aligned}
$$


where $\left\{T^{a}, T^{b}\right\}=T^{a}\left(T^{b}\right)+T^{b}\left(T^{a}\right)$. The induced contravariant metric associated to this operator is computed to be the following positive definite matrix

$$
g^{(i j)}=\left(\begin{array}{ccc}
\left(x^{2}+1\right)^{2} & 0 & \left(x^{2}+1\right)\left(z^{2}+1\right) \\
0 & y^{4}+4 y^{2}+1 & 0 \\
\left(x^{2}+1\right)\left(z^{2}+1\right) & 0 & 2\left(z^{2}+1\right)^{2}
\end{array}\right),
$$

whose determinant is $g=\left(x^{2}+1\right)^{2}\left(y^{4}+4 y^{2}+1\right)\left(z^{2}+1\right)^{2}$. Then, with respect to this nondegenerate metric, the operator $\mathcal{H}$ can also be described as

$$
-2 \mathcal{H}=\Delta+\vec{V}+U,
$$

where $\vec{V}=-2\left(x^{3}+x+z+z x^{2}\right) p-2\left(2 y+y^{3}\right) q-2\left(2 z^{3}+2 z+x+x z^{2}\right) r$. It is not hard to verify, always with respect to the metric (4), that the first order term $\vec{V}$ is the gradient of the function $\lambda=-\ln \left(x^{2}+1\right)-1 / 2 \ln \left(y^{4}+4 y^{2}+1\right)-\ln \left(z^{2}+1\right)$. Hence, by considering the gauge factor

$$
\mu=e^{\frac{\lambda}{2}}=\left(x^{2}+1\right)^{-1 / 2}\left(y^{4}+4 y^{2}+1\right)^{-1 / 4}\left(z^{2}+1\right)^{-1 / 2},
$$

the operator $\mathcal{H}$ is gauge equivalent to a Schrödinger operator

$$
-2 \mathcal{H}_{0}=\Delta+U
$$

were the potential is a rational function of $y$. Furthermore, it is not hard to show that, after the gauge transformation, the functions in $\widetilde{\mathcal{N}}_{111}=\left\{\mu \cdot x^{i} y^{j} z^{k} \mid 0 \leq i, j, k \leq 1\right\}$ are square integrable with respect to $\sqrt{g} d x d y d z$. Recall here that $g$ is the determinant of the covariant metric, hence $\sqrt{g}=\mu^{2}$. Thus, for $i, j, k$ either 0 or 1 , one can use Fubini's theorem to decompose the integral

$$
\iiint_{\mathbb{R}^{3}}\left(\mu x^{i} y^{j} z^{k}\right)^{2} \mu^{2} d x d y d z=\iiint_{\mathbb{R}^{3}} \frac{x^{2 i} y^{2 j} z^{2 k}}{\left(x^{2}+1\right)^{2}\left(y^{4}+4 y^{2}+1\right)\left(z^{2}+1\right)^{2}} d x d y d z,
$$

into the product of three finite integrals in one variable. Consequently the operator $\mathcal{H}_{0}$ is a normalizable quasi-exactly solvable Schrödinger operator and it is possible to compute eight eigenfunctions by diagonalizing the matrix obtained by restricting $\mathcal{H}$ to $\mathcal{N}$. For this operator, one gets two eigenvalues, -3 and 1 , both of multiplicity four. The eight eigenfunctions associated to these two eigenvalues are respectively,

$$
\begin{aligned}
& \psi_{-3,1}=-1+x z, \quad \psi_{-3,2}=y-x y z, \quad \psi_{-3,3}=x y+y z, \quad \psi_{-3,4}=x+z, \\
& \psi_{1,1}=y+x y z, \quad \psi_{1,2}=-x+z, \quad \psi_{1,3}=-x y+y z, \quad \psi_{1,4}=1+x z .
\end{aligned}
$$

Finally, one gets eight eigenfunctions of the Schrödinger operator $\mathcal{H}_{0}$ by scaling each of these functions by the gauge factor $\mu$.

In general, there is no a-priori method for testing whether a given differential operator is Lie algebraic or quasi-exactly solvable. However, one can try to perform a classification of these operators under local equivalence using the four-step general method of classification described by González-López, Kamran and Olver in [11].

The first step toward the classification of normalizable quasi-exactly solvable Schrödinger operators is to classify the finite dimensional Lie algebras of first order differential operators up to diffeomorphism and rescaling. Then, the task is to determine which of these equivalence classes admit a finite dimensional $\mathfrak{g}$-module $\mathcal{N}$ of smooth functions. Then, from the quasiexactly solvable Lie algebras found in the second step, one can construct second order differential operator as described in (1) from any choice of coefficients $C_{a b}, C_{c}$, and $C_{0}$. The third step consists to determine which of these operators are equivalent to Schrödinger operators and this 
can be performed by verifying the closure condition. Finally, the last step in this classification problem is to check if the functions contained in the $\widetilde{\mathfrak{g}}$-module $\widetilde{\mathcal{N}}$ are square integrable.

As mentioned previously, the entire classification has been established in one dimension. In the scope of the first two steps, every quasi-exactly solvable Lie algebra is locally equivalent to a subalgebra of the Lie algebra

$$
\mathfrak{g}_{n}=\operatorname{Span}\left\{\frac{\partial}{\partial x}, x \frac{\partial}{\partial x}, x^{2} \frac{\partial}{\partial x}-n z, 1\right\},
$$

where $n$ is a non negative integer, see [13] for more details. Then, once a second order differential operator is constructed, since all one forms are closed in one dimension, such operator will always be equivalent to a Schrödinger operator, reducing the third step to a trivial step. Finally González-López, Kamran and Olver determined in [10] necessary and sufficient conditions for the normalizability of the eigenfunctions of the quasi-exacly solvable Schrödinger operators.

In two dimensions, the first two steps of the classification problem were determined by the same authors in [12] and [14]. Based upon Lie's classification of Lie algebras of vector fields, see [18], a complete classification of the quasi-exactly solvable Lie algebras $\mathfrak{g}$ of first order differential operators, together with their finite dimensional $\mathfrak{g}$-modules, was completed. The case of two complex variables is discussed in the first two papers while the third paper completed the classification by considering operators on two real variables. However, the last two steps are not yet completed but a wide variety of normalizable quasi-exactly solvable Schrödinger operators has been exhibited, see for instance [11, 13] and [14].

In the next section, a partial classification of quasi-exactly solvable Lie algebras of first order differential operators in three dimensions is given. While these two first steps were successfully completed in one and two dimensions, only part of this work is now done in three dimensions. However, these new quasi-exactly solvable Lie algebras can be used to seek new quasi-exactly solvable Schrödinger operators in three dimensional space. The last section of this paper is devoted to the description of new quasi-exactly solvable Schrödinger operators in three dimensions. Eigenvalues are also computed for two families of Schrödinger operators. These eigenvalues are part of the spectrum of the operators and their eigenfunctions, together with their nodal surfaces, are exhibited. In addition, a connection is made between the separability theorem proved in [6] and the quasi-exactly solvable Schrödinger operators on flat manifold. The quasi-exactly solvable models obtained in our paper are new as far as we can tell. In particular they are not part of the list of multi-dimensional quasi-exactly solvable models obtained in [26] by the method of inverse separation of variables.

\section{Classification of quasi-exactly solvable Lie algebras of first order differential operators}

\subsection{Lie algebras of first order differential operators}

Our goal in this section is to give a partial classification of quasi-exactly solvable Lie algebras of first order differential operators in three dimensions. A first step toward this goal is to obtain a classification of the finite dimensional Lie algebras $\mathfrak{g}$ of first order differential operators. After this is done, the next step is to impose the existence of an explicit finite dimensional $\mathfrak{g}$ module $\mathcal{N}$ of smooth functions. To this end, we will first summarize the basic theory underlying the classification of Lie algebras of first order differential operators.

For $\mathbf{M}$ an $n$-dimensional manifold, we denote by $\mathcal{F}(\mathbf{M})$ the space of smooth real-valued functions and $\mathcal{V}(\mathbf{M})$ the Lie algebra of vector fields on $\mathbf{M}$. The space $\mathcal{F}(\mathbf{M})$ form a $\mathcal{V}(\mathbf{M})$ module under the usual derivation $\eta \rightarrow v(\eta)$, where $v$ is a vector field in $\mathcal{V}(\mathbf{M})$ and $\eta$ a function in $\mathcal{F}(\mathbf{M})$. The Lie algebra of first order differential operators $\mathcal{D}^{1}(\mathbf{M})$ can be described as 
a semidirect product of these two spaces, $\mathcal{D}^{1}(\mathbf{M})=\mathcal{V}(\mathbf{M}) \ltimes \mathcal{F}(\mathbf{M})$. Indeed, each element $T$ in $\mathcal{D}^{1}(\mathbf{M})$ can be written into a sum $T=v+\eta$ and the Lie bracket is given by

$$
\left[T^{1}, T^{2}\right]=\left[v^{1}, v^{2}\right]+v^{1}\left(\eta^{2}\right)-v^{2}\left(\eta^{1}\right), \quad \text { where } \quad T^{i}=v^{i}+\eta^{i} \in \mathcal{D}^{1}(\mathbf{M}) .
$$

Note that the space $\mathcal{F}(\mathbf{M})$ is also a $\mathcal{D}^{1}(\mathbf{M})$-module with $T(\zeta)=v(\zeta)+\eta \cdot \zeta$. Consequently, any finite dimensional Lie algebra of first order differential operators $\mathfrak{g}$ can be written as

$$
T^{1}=v^{1}+\eta^{1}, \quad \ldots, \quad T^{s}=v^{s}+\eta^{s}, \quad T^{s+1}=\zeta^{1}, \quad \ldots, \quad T^{s+r}=\zeta^{r},
$$

where $v^{1}, \ldots, v^{s}$ are linearly independent vector fields spanning $\mathfrak{h} \subset \mathcal{V}(\mathbf{M})$, a $s$-dimensional Lie algebra and where the functions $\zeta^{1}, \ldots, \zeta^{r}$ act as multiplication operators and span $\mathcal{M} \subset \mathcal{F}(\mathbf{M})$ a finite dimensional $\mathfrak{h}$-module. Note that restrictions need to be imposed to the functions $\eta^{i}$ for $\mathfrak{g}$ to be a Lie algebra. Indeed, without the cohomological conditions that will be described below, the Lie bracket given in (5) does not necessarily return an element in the Lie algebra $\mathfrak{g}$.

For $T=v+\eta$, we define a 1-cochain $F: \mathfrak{h} \rightarrow \mathcal{F}(\mathbf{M})$ by the linear map $\langle F ; v\rangle=\eta$. Since any function $\zeta \in \mathcal{M}$ can be added to $T$ without changing the Lie algebra $\mathfrak{g}$, this map is not well defined. To deal with this issue, we should therefore interpret $F$ as a $\mathcal{F}(\mathbf{M}) / \mathcal{M}$-valued 1-cochain. Thus, from the Lie bracket given in (5), it is straightforward to see that $\mathfrak{g}$ is a Lie algebra if and only if the 1-cochain $F$ satisfies the bilinear identity

$$
v^{i}\left\langle F ; v^{j}\right\rangle-v^{j}\left\langle F ; v^{i}\right\rangle-\left\langle F ;\left[v^{i}, v^{j}\right]\right\rangle \in \mathcal{M}, \quad v^{i}, v^{j} \in \mathfrak{h} .
$$

In terms of Lie algebra cohomology, this condition can be restated as follow, $\left\langle\delta_{1} F ; v^{i}, v^{j}\right\rangle \in \mathcal{M}$ for all $v^{i}, v^{j}$ in $\mathfrak{h}$, i.e. $F$ is a $\mathcal{F}(\mathbf{M}) / \mathcal{M}$-valued 1-cocycle on $\mathfrak{h}$. (See [7] for a detailed description of Lie algebra cohomology.)

This classification of Lie algebras of first order differential operators would not be complete without considering the local equivalences between the Lie algebras. Indeed, if a gauge transformation with gauge factor $\mu=e^{\lambda}$, is performed on an operator $T=v+\eta$ in $\mathfrak{g}$, the resulting differential operator $\widetilde{T}=e^{\lambda} \cdot T \cdot e^{-\lambda}=v+\eta-v(\lambda)$ will only differ from $T$ by the addition of a multiplication operator $v(\lambda)$. Again, this can be expressed in cohomological terms. Indeed, under the 0-coboundary map $\delta_{0}: \mathfrak{h} \rightarrow \mathcal{F}(\mathbf{M}) / \mathcal{M}$ defined by $\left\langle\delta_{0} \lambda ; v\right\rangle=v(\lambda)$, the multiplication factor $v(\lambda)$ can be interpreted as the image, or the 0-coboundary, of the function $\lambda$. Hence, combining these two observations, it is possible to conclude that the map $F$ is an element in $H^{1}(\mathfrak{h}, \mathcal{F}(\mathbf{M}) / \mathcal{M})=\operatorname{ker} \delta_{1} / \operatorname{Im} \delta_{0}$. Thus, if two differential operators $\mathfrak{g}$ and $\widetilde{\mathfrak{g}}$ are equivalent with respect to a change of variables $\varphi$ and a gauge transformation given by $\mu=e^{\lambda}$, these two operators will correspond to equivalent triples $(\mathfrak{h}, \mathcal{M},[F])$, and $(\widetilde{\mathfrak{h}}, \widetilde{\mathcal{M}},[\widetilde{F}])$, where $\widetilde{\mathfrak{h}}=\varphi_{*}(\mathfrak{h})$, $\widetilde{\mathcal{M}}=\varphi_{*}(\mathcal{M})$, and $\widetilde{F}=\varphi_{*} \circ F \circ \varphi_{*}^{-1}+\delta_{0} \lambda$. This is summarized in the following theorem.

Theorem 1. There is a one to one correspondence between equivalence classes of finite dimensional Lie algebras $\mathfrak{g}$ of first order differential operators on $\mathbf{M}$ and equivalence classes of triples $(\mathfrak{h}, \mathcal{M},[F])$, where

1) $\mathfrak{h}$ is a finite dimensional Lie algebra of vector fields;

2) $\mathcal{M}$ is a finite dimensional $\mathfrak{h}$-module of functions;

3) $[F]$ is a cohomology class in $H^{1}(\mathfrak{h}, \mathcal{F}(\mathbf{M}) / \mathcal{M})$.

Hence the general classification of finite dimensional Lie algebras of first order differential operators $\mathfrak{g}$ can be bring down to the classification of triples $(\mathfrak{h}, \mathcal{M},[F])$ under local changes of variables.

In three dimensions, a complete local classification of the finite dimensional Lie algebras of vector fields $\mathfrak{h}$ has been established by Lie in [18] and Amaldi in [1]. Lie's classification 
distinguishes between the imprimitive Lie algebras, for which their exists an invariant foliation of the manifold, and the primitive Lie algebras, for which no such foliation exists. Lie's work gives a description of the eight different classes of primitive Lie algebras and, based under the possible foliations of the manifold, the imprimitive Lie algebras are subdivided into the following three types:

I. The manifold admits locally an invariant foliation by surfaces that does not decompose into a foliation by curves.

II. The manifold admits locally an invariant foliation by curves not contained in a foliation by surfaces.

III. The manifold admits locally an invariant foliation by surfaces that does decompose into a foliation by curves.

Observe that these three types are not necessarily exclusive. For instance, the Lie algebra $\mathfrak{h}=\{p, q, x q, x p-y q, y p, r\}$ belongs to the first two types. The underlying manifold $\mathbb{R}^{3}$ admits a first indecomposable foliation by planes $\Delta:=\{z=$ constant $\}$ and also admits an second invariant foliation by straight lines $\Phi:=\{x=$ constant $\} \cap\{y=$ constant $\}$ not contained in any invariant surfaces. Lie classified the algebras of type I and II, giving respectively twelve and twenty-one different classes of Lie algebras. Few years latter, the 103 classes of Lie algebras of the third type were exhibited by Amaldi.

The number of finite dimensional Lie algebras of vector fields $\mathfrak{h}$ is large and it did not seem reasonable to consider all the 154 classes. For this first classification attempt, we have chosen to focus on the algebras which seem promising in our aim to construct new quasi-exactly solvable Schrödinger operators. The selection was made upon the following criteria.

We first narrowed our choice based on the results given in [6]; provided the Lie algebra $\mathfrak{g}$ is imprimitive and its invariant foliation consists of surfaces, one can show, adding some other hypothesis on the metric induced, that a Lie algebraic Schrödinger operator generated by $\mathfrak{g}$ separates partially in either Cartesian, cylindrical or spherical coordinates. Since such algebras are good candidates for generating interesting quasi-exactly solvable Schrödinger operators, we restricted our search on the type I and type III imprimitive algebras. In this paper, the classification of the twelve type I Lie algebras is entirely performed while, for the type III Lie algebras, we focused on some of the most general Lie algebras. Since the induced metric $g^{(i j)}$ needs to be non-degenerate, the type III Lie algebras involving only one or two of the three partial derivatives were discarded. Finally we selected our algebras among those that contain other type III algebras as subalgebras.

\subsection{Classification of Lie algebras of first order differential operators}

Using the equivalence between the Lie algebras of first order differential operators $\mathfrak{g}$ and triples $(\mathfrak{h}, \mathcal{M},[F])$, it is possible to determine the Lie algebras $\mathfrak{g}$ from the selected Lie algebras of vector fields $\mathfrak{h}$. But first, recall that the second step in the classification of quasi-exactly Schrödinger operators is to determine which of these Lie algebras of first order differential operators $\mathfrak{g}$ are quasi-exactly solvable. It is not hard to see that if $\mathfrak{g}$ is quasi-exactly solvable with non trivial fixed module $\mathcal{N}$, the Lie algebra $\mathfrak{g}$ is finite dimensional if and only if $\mathcal{M}$ is the module of constant functions, see [12] for details. Therefore, instead of working on the general classification of Lie algebras of first order differential operators $\mathfrak{g}$, we will restrict our work to the equivalence classes of triples $(\mathfrak{h},\{1\},[F])$. Thus, for each of the selected Lie algebras $\mathfrak{h}$, we first seek for the possible cohomology classes, $[F]$ in $H^{1}(\mathfrak{h}, \mathcal{F}(\mathbf{M}) /\{1\})$. Once this is done, in the scope of the second step, it will be left to find if there exists an explicit finite dimensional $\mathfrak{g}$-module $\mathcal{N}$, where $\mathfrak{g}$ is the Lie algebra equivalent to the triple $(\mathfrak{h},\{1\},[F])$. Note that, as in lower dimensions, the existence of a nontrivial module $\mathcal{N}$ will impose a "quantization" condition on $[F]$. Indeed, for each of the 
Lie algebras worked out in this paper, the possible values for the functions in $[F]$ can only be taken in a discrete set. For detailed results related to the quantization of cohomology, see [8] and [22].

\subsubsection{Classification of the cohomology classes $[F]$ in $H^{1}(\mathfrak{h}, \mathcal{F}(\mathrm{M}) /\{1\})$}

To determine the possible cohomology classes, we first start with $[F]$ as general as possible. For every $v$ in the Lie algebra $\mathfrak{h}$, we denote the value of the 1-cocycle $\langle F ; v\rangle$ by $\eta_{v}$, and $\eta_{v}$ can be any function in $\mathcal{F}(\mathbf{M}) /\{1\}$. Our aim is to find the most general 1-cocycle $F$, that is the most general functions $\eta_{v}$, satisfying the restrictions imposed by the 1-cocycle conditions (7). Then, using the 0 -coboundary map, we try to describe the class $[F]$ with representatives $\eta_{v}$ as simple as possible. Finally, if $\left\{v^{1}, \ldots, v^{r}\right\}$ is a basis for $\mathfrak{h}$, the set $\left\{v^{1}+\eta_{v^{1}}, \ldots, v^{r}+\eta_{v^{r}}\right\}$ will be a basis for the Lie algebra $\mathfrak{g}$. Note that in this process, one can alternate the use of the 1-cocycle restrictions with the use of the 0-coboundary cancellations. For instance, if the element $p$ belongs to the algebra $\mathfrak{h}$, the function $\langle F ; p\rangle=\eta_{p}$ can be annihilated by the image of the function $\Psi_{p}=\int \eta_{p} d x$ under the 0-coboundary map. Indeed $\left\langle\delta_{0} \Psi_{p} ; p\right\rangle=p\left(\int \eta_{p} d x\right)=\eta_{p}$ and $\widetilde{F}=F-\delta_{0} \Psi_{p}$ belong to $[F]$. Thus, we can assume the function $\eta_{p}$ to be equivalent to the zero function. Then for another vector field $v$ in $\mathfrak{h}$, using the 1-cocycle restriction for the pair $(p, v)$, that is

$$
p\langle F ; v\rangle-v\langle F ; p\rangle-\langle F ;[p, v]\rangle=p \eta_{v}-0-\eta_{[p, v]} \in\{1\}
$$

one obtains conditions on the two functions $\eta_{v}$ and $\eta_{[p, v]}$. Once again, one might try to absorb part of the function $\eta_{v}$ with $\delta_{0} \Psi_{v}$, the image of another function $\Psi_{v}$. Note that, in order to maintain $\eta_{p} \equiv 0$, a restriction is imposed on $\Psi_{v}$. Indeed, when the 1 -cocycle $F+\delta_{0} \Psi_{v}$ is applied to $p$, we have to avoid reintroducing a function for $\eta_{p}$. Thus we need to consider only the functions $\Psi_{v}$ for which $\left\langle\delta_{0} p ; \Psi_{v}\right\rangle=\left(\Psi_{v}\right)_{x}$ is a constant function. Then, to complete the determination of $[F]$, the same process is preformed to every vector field of $\mathfrak{h}$, with some care in the choices of the 0 -coboundary maps, avoiding to undo the simplifications done in the previous steps.

The results of this partial classification of cohomology classes $[F]$, that gives a partial classification of Lie algebras of differential operators $\mathfrak{g}$, are summarized in Tables 1 and 2 at the end of this section. The first table gives a 1-cocycle representative for the twelve type I Lie algebras and Table 2 exhibits the results for some general Lie algebras of vector fields among the type III Lie algebras. For these two tables, the classification numbers, given respectively by Lie and Amaldi, sit in the the first column. The second column gives a basis for the Lie algebra $\mathfrak{h}$ and the third column exhibits the first order differential operators $v+\eta_{v}$ for which $\langle F ; v\rangle=\eta_{v}$ in not trivial. $\eta_{v}$ is taken to be the simplest representative and when the function $\eta_{v}$ is trivial, the differential operator is simply the vector field exhibited in the second column.

It would be impractical to present the details of the computations in all cases. Furthermore, the arguments are quite similar for all Lie algebra $\mathfrak{h}$ of vector fields. So, for brevity's sake, we will only give the details for two of the selected Lie algebras. The chosen examples illustrate well the general process and will give to the reader a good idea of how the calculations proceed in general.

Type I, case 1. This Lie algebra $\mathfrak{h}$ is spanned by the eight vector fields $p, q, x p, y q, x q$, $y p, x^{2} p+x y q$ and $x y p+y^{2} q$. The vector field $p$ belongs to the Lie algebra, hence, as mentioned previously, the function $\eta_{p}$ can be assumed to be zero. The 1-cocycle condition for the pair $(p, q)$ imposes the following restriction

$$
\left\langle\delta_{1} F ; p, q\right\rangle=\left(\eta_{q}\right)_{x}-\left(\eta_{p}\right)_{y}-\langle F ;[p, q]\rangle=\left(\eta_{q}\right)_{x} \in\{1\} .
$$

Thus $\eta_{q}=c_{q} x+h_{q}(y, z)$, where $c_{q}$ is a constant. Hopefully, the function $h_{q}(y, z)$ can be absorbed by the image, under the 0-coboundary map, of the function $\Psi_{q}=\int h_{q}(y, z) d y$. Since $\left(\Psi_{q}\right)_{x}$ is zero, the 0 -coboundary of $\Psi_{q}$ will not affect $\eta_{p}$. 
Similarly, by considering the pair $(p, x p)$, one concludes that $\eta_{x p}=c_{x p} x+h_{x p}(y, z)$, where $c_{x p} x$ can be canceled, without changing the previous functions, by the 0-coboundary of the function $\Psi_{x p}=c_{x p} x$. Then, for the pair $(q, x p)$, the restriction reads as

$$
\left\langle\delta_{1} F ; q, x p\right\rangle=\left(\eta_{x p}\right)_{y}-x\left(\eta_{q}\right)_{x}-\langle F ;[q, x p]\rangle=\left(h_{x p}(y, z)\right)_{y}-x \cdot c_{q} \in\{1\} .
$$

Necessarily, since $h_{x p}$ depends only on $y$ and $z$, the constant $c_{q}$ has to be zero and the function $h_{x p}(y, z)$ is forced to be of the form $d_{x p} y+K(z)$, where $d_{x p}$ is a constant. Thus, at this point, $\eta_{p}=0, \eta_{q}=0$ and $\eta_{x p}=d_{x p} y+K(z)$.

Consider now the three vector fields $y q, x q$ and $y p$. If we pair each of them with $p$ and $q$, from the six 1-cocycle restrictions, one obtains directly the following

$$
\eta_{y q}=c_{y q} x+d_{y q} y+k_{y q}(z), \quad \eta_{x q}=c_{x q} x+d_{x q} y+k_{x q}(z), \quad \eta_{y p}=c_{y p} x+d_{y p} y+k_{y p}(z) .
$$

With the image of the function $\Psi_{y q}=d_{y q} y$, the function $\eta_{y q}$ can be reduced to $\eta_{y q}=c_{y q} x+k_{y q}(z)$ without undoing the previous work. From the restriction associated to the pair $(x p, y p)$, one easily check that

$$
\left\langle\delta_{1} F ; x p, y p\right\rangle=x\left(\eta_{y p}\right)_{x}-y\left(\eta_{x p}\right)_{x}+\eta_{y p}=x \cdot c_{y p}+c_{y p} x+d_{y p} y+k_{y p}(z) \in\{1\},
$$

forcing $c_{y p}$ and $d_{y p}$ to be zero and $k_{y p}(z)$ to be a constant function. Similarly, by considering the pair $(x p, y q)$, one obtains that the function $x \cdot c_{y q}-y \cdot d_{x p}$ must be constant, hence $c_{y q}$ and $d_{x p}$ are zero. To completely determine the functions $\eta_{v}$ for these three vector fields, two restrictions, associated to the pairs $(x p, x q)$ and $(y p, x q)$, must be verified. The first imposes that $x\left(\eta_{x q}\right)_{x}-x\left(\eta_{x p}\right)_{y}-\eta_{x q}=x \cdot c_{x q}-c_{x q} x-d_{x q} y-k_{x q}(z)$ must be constant. Thus it leaves no choice but to take $d_{x q}$ as the constant zero and $k_{x q}(z)$ as a constant function. Finally, the last restriction forces $y\left(\eta_{x q}\right)_{x}-x\left(\eta_{y p}\right)_{y}-\eta_{y q}+\eta_{x p}=y \cdot c_{x q}-k_{y q}(z)+K(z)$ to be a constant, hence $c_{x q}$ must be zero while $k_{x q}(z)$ must be equal, modulo the constant functions, to the function $K(z)$. Putting together these restrictions, the image of the 1-cocycle $F$ for the first six vector fields of $\mathfrak{h}$ can be described as $\eta_{p}=\eta_{q}=\eta_{x q}=\eta_{y p}=0$ and $\eta_{x p}=\eta_{y q}=K(z)$. One easily checks that the remaining two restrictions are satisfied.

To determine completely the 1-cocycle $F$, it remains to find its images for the two vector fields $T:=x^{2} p+x y q$ and $Q=x y p+y^{2} q$. For $\eta_{T}$, three restrictions are needed to reach that $\eta_{T}=3 x K(z)$. Indeed, from the pair $(p, T)$, the cocycle condition forces the following equality $\left(\eta_{T}\right)_{x}-2 \eta_{x p}-\eta_{y q}=c_{T}$, where $c_{T}$ is a constant. It is not hard to see that $\eta_{T}$ must be equal to $3 x K(z)+c_{T} x+h_{T}(y, z)$. From the pair $(q, T)$, we get similarly that $\eta_{T}=3 x K(z)+c_{T} x+$ $d_{T} y+k_{T}(z)$. Finally, the restriction for the pair $(x p, T)$ leads to

$$
x\left(\eta_{T}\right)_{x}-T\left(\eta_{x p}\right)-\eta_{T}=x \cdot 3 K(z)+x \cdot c_{T}-\left(3 x K(z)+c_{T} x+d_{T} y+k_{T}(z)\right) \in\{1\} .
$$

Hence the constant $d_{T}$ dies out and $k_{T}(x)$ has to be a constant function. Note that, with these 3 restrictions, $\eta_{T}=3 x\left(K(z)+c_{T} / 3\right)$, but, by taking $\eta_{x p}=K(z)+c_{T} / 3$, one gets the claimed result. By symmetry on $x$ and $y$, the exact same arguments lead to $\eta_{Q}=3 y K(z)$. It is then straightforward to verify that the 1-cocycle $F$, given by the eight functions

$$
\eta_{p}=\eta_{q}=\eta_{x q}=\eta_{y p}=0, \quad \eta_{x p}=\eta_{y q}=K(z), \quad \eta_{P}=3 x K(z) \quad \text { and } \quad \eta_{Q}=3 y K(z),
$$

satisfies all the other 1-cocycle conditions. Finally, the Lie algebra $\mathfrak{g}$ associated to this triple $(\mathfrak{h},\{1\},[F])$ is the Lie algebra spanned by

$$
\left\{p, q, x p+K(z), y p, x q, y q+K(z), x^{2} p+x y q+3 x K(z), x y p+y^{2} q+3 y K(z), 1\right\},
$$

where $K(z)$ can be any function.

Fortunately the calculations performed for a given Lie algebra $\mathfrak{h}$ can be repeated for any other Lie algebra sharing a subset of generators with $\mathfrak{h}$. Note also that some ad hoc lemma's were used trough this work to simplify these calculations. For instance. 
Lemma 1. Let $i: \mathbb{R}^{2} \rightarrow \mathbb{R}^{3},(x, y) \mapsto(x, y, z)$ denote the inclusion map and suppose that $\mathfrak{h}_{0} \subset \Gamma\left(i_{*} T \mathbb{R}^{2}\right)$, meaning that the generators of $\mathfrak{h}_{0}$ depend on the variables $x$ and $y$ only. Let $\mathfrak{h}$ be a Lie algebra of vector fields on $\mathbb{R}^{3}$ given by $\mathfrak{h}=\mathfrak{h}_{0} \oplus\left\{r, z r, z^{2} r\right\}$. If, for non constant functions $f(x, y)$ and $g(x, y)$, the vector fields $f(x, y) p$ and $g(x, y) q$ belong to $\mathfrak{h}$ and if their associated images $\eta_{f(x, y) p}$ and $\eta_{g(x, y) q}$ depend on $x$ and $y$ only, then

$$
H^{1}\left(\mathfrak{h}, \mathcal{F}\left(\mathbb{R}^{3}\right) /\{1\}\right)=H^{1}\left(\mathfrak{h}_{0}, \mathcal{F}\left(\mathbb{R}^{2}\right) /\{1\}\right) \oplus H^{1}\left(\left\{r, z r, z^{2} r\right\}, \mathcal{F}(\mathbb{R}) /\{1\}\right)
$$

Proof. Denote $A:=f(x, y) p$ and $B:=g(x, y) q$. From the cocycle restrictions associated to the pairs $\left(A, z^{i} r\right)$, where $i=0,1,2$, we obtain

$$
\begin{aligned}
\left\langle\delta_{1} F ; A, z^{i} r\right\rangle & =A\left(\eta_{z^{i} r}\right)-z^{i}\left(\eta_{A}\right)_{z}-\left\langle F ;\left[A, z^{i} r\right]\right\rangle=f(x, y)\left(\eta_{z^{i} r}\right)_{x}-0-\langle F, 0\rangle \\
& =f(x, y)\left(\eta_{z^{i} r}\right)_{x} \in\{1\} .
\end{aligned}
$$

Since $f(x, y)$ is not constant, $\left(\eta_{z^{i} r}\right)_{x}$ must vanish, hence the functions $\eta_{z^{i} r}$ depend on $y$ and $z$. In a similar way, from the restrictions associated to the pairs $\left(B, z^{i} r\right)$ it is straightforward to conclude that $\eta_{z^{i} r}=h^{i}(z)$. Finally, for any element $v$ in $\mathfrak{h}_{0}$, the function $\eta_{v}$ will depend on $x$ and $y$ only. Indeed, since $\eta_{z r}$ depends on $z$ only,

$$
\left\langle\delta_{1} F ; v, z r\right\rangle=v\left(\eta_{z r}\right)-z\left(\eta_{v}\right)_{z}-\langle F ;[v, z r]\rangle=0-z\left(\eta_{v}\right)_{z}-\langle F, 0\rangle=-z\left(\eta_{v}\right)_{z} \in\{1\} .
$$

Therefore $\left(\eta_{v}\right)_{z}$ must be zero, forcing the function $\eta_{v}$ to depend on $x$ and $y$ only.

Note that $H^{1}\left(\left\{r, z r, z^{2} r\right\}, \mathcal{F}(\mathbb{R}) /\{1\}\right)$ is already well known. The 1-cocycle $F$ associated the Lie algebra $\mathfrak{h}=\left\{r, z r, z^{2} r\right\}$ is determined by three functions and the simplest representative is given by $\eta_{r}=0, \eta_{z r}=0$ and $\eta_{z^{2} r}=d z$, for any constant $d$. Thus, one can use this lemma to simplify some of the computations required in this classification problem. For instance, given $\mathfrak{h}$ the type I Lie algebra of vector fields given by case 10 in Table 1, the Lie algebra of differential operators $\mathfrak{g}$ built from $\mathfrak{h}$ is obtained from a direct application of this lemma.

Type I, case 10. The Lie algebra $\mathfrak{h}=\operatorname{Span}\left\{p, q, x p, y q, x q, y p, x^{2} p+x y q, x y p+y^{2} q, r, z r, z^{2} r\right\}$ can be decomposed as $\mathfrak{h}_{0} \oplus\left\{r, z r, z^{2} r\right\}$ where $\mathfrak{h}_{0}$ is the case 1 Lie algebra from the same table. It was shown in the previous calculations that the functions $\eta_{y p}$ and $\eta_{x q}$ are zero, hence functions on $x$ and $y$ only. Thus the case 10 Lie algebra, along with its two vector fields $y p$ and $x q$, satisfies the requirements of the Lemma 1 . Therefore, for the vector fields in the algebra $\mathfrak{h}_{0}$, the values of the 1-cocycle depend on $x$ and $y$ only, forcing $K(z)$ to be $c$ a constant function. It is then obvious that the 1-cocycle $F$ is defined by eleven functions, were the three non-zero are given by $\eta_{T}=c x, \eta_{Q}=c y$ and $\eta_{z^{2} r}=d x$, for $c$ and $d$ any constants. The Lie algebra of first order differential operators $\mathfrak{g}$ corresponding to this triple is then

$$
\mathfrak{g}=\operatorname{Span}\left\{p, q, x p, y q, x q, y p, x^{2} p+x y q+c x, x y p+y^{2} q+c y, r, z r, z^{2} r+d z, 1\right\} .
$$

It should be pointed here that $H^{1}(\mathfrak{h}, \mathcal{F}(\mathbf{M}) /\{1\})$ and $H^{1}(\mathfrak{h}, \mathcal{F}(\mathbf{M}))$ can also be determined alternatively using isomorphisms given in [20] and [21]. For the case that interests us, that is $H^{1}(\mathfrak{h}, \mathcal{F}(\mathbf{M}) /\{1\})$, we fix a base point $e$ and denote $\mathfrak{i}$ the isotropy subalgebra. Provided the existence of a subalgebra $\mathfrak{a} \subset \mathfrak{h}$ which is complementary to $\mathfrak{i}$, one can show, see [20], that

$$
H^{1}(\mathfrak{h}, \mathcal{F}(\mathbf{M}) /\{1\}) \cong H^{2}(\mathfrak{h} / \mathfrak{i})
$$

This isomorphism leads to an explicit method for constructing 1-cocycle representatives $F$ in $H^{1}(\mathfrak{h}, \mathcal{F}(\mathbf{M}) /\{1\})$. We first choose $\alpha$, a 2-cocycle representative of a class in $H^{2}(\mathfrak{h} / \mathfrak{i})$, and, for $\left\{v^{1}, \ldots, v^{n}\right\}$ a basis of $\mathfrak{h}$, we denote $\alpha_{i j}=\alpha\left(v^{i}, v^{j}\right)$. If $\mathfrak{a}=\left\{v^{1}, \ldots, v^{m}\right\}, \mathfrak{i}=\left\{v^{m+1}, \ldots, v^{n}\right\}$, 
and $c_{i j}^{k}$ are the structure constants of the Lie algebra $\mathfrak{h}$, a 1-cocycle in $H^{1}(\mathfrak{h}, \mathcal{F}(\mathbf{M}) /\{1\})$ will be obtained by solving first the following $m(m-1)$ equations

$$
v^{i}\left(f_{j}\right)-v^{j}\left(f_{i}\right)-\sum_{k} c_{i j}^{k} f_{k}=\alpha_{i j}, \quad \text { for } \quad 1 \leq i<j \leq m
$$

Once a non unique solution $f_{1}, \ldots, f_{m}$ is obtained, the remaining functions $f_{m+1}, \ldots, f_{n}$ are determined as the unique solution to the $m(n-m)$ equations

$$
v^{i}\left(f_{j}\right)-v^{j}\left(f_{i}\right)-\sum_{k} c_{i j}^{k} f_{k}=\alpha_{i j}, \quad \text { for } \quad 1 \leq i \leq m, \quad m+1 \leq j \leq n,
$$

with initial conditions

$$
f_{i}(e)=0 \quad \text { for } \quad m+1 \leq i \leq n .
$$

Note however that this method can not be applied to all the three dimensional Lie algebras since the existence of the complementary Lie subalgebra is not guaranteed. For instance, the type III case $17 \mathrm{~A}_{1}$ can not be treated using the isomorphism. Indeed, for the Lie algebra

$$
\mathfrak{h}=\left\{p, q, x p+z r, y q, x^{2} p+(2 x+a z) z r, y^{2} q\right\},
$$

and the base point $e=(0,0,0)$, the isotropy algebra $i$ is generated by the last four elements and the algebra $\mathfrak{a}=\{p, q\}$ fails to be complementary, due to the absence of the element $r$ in the Lie algebra. One can easily verify that $Z^{2}(\mathfrak{h} / \mathfrak{i})=\left\{\alpha_{1} \wedge \alpha_{3}, \alpha_{1} \wedge \alpha_{5}, \alpha_{2} \wedge \alpha_{4}, \alpha_{2} \wedge \alpha_{6}\right\}$ and $B^{2}(\mathfrak{h} / \mathfrak{i})=\left\{\alpha_{1} \wedge \alpha_{3}, \alpha_{2} \wedge \alpha_{4}\right\}$. One can observe at that point that the theorem does not hold, since the dimension of $H^{2}(\mathfrak{h} / \mathfrak{i})$ is two while the dimension of $H^{1}(\mathfrak{h}, \mathcal{F}(\mathbf{M}) /\{1\})$ was computed to be three previously. Moreover applying the technique to the 2-cocycle $\alpha=c \cdot \alpha_{1} \wedge \alpha_{5}+d \cdot \alpha_{2} \wedge \alpha_{6}$, one gets a 1-cocycle that does not satisfies all the conditions that were not considered in the technique detailed above.

\subsection{Classification of quasi-exactly solvable Lie algebras of first order differential operators and the quantization condition}

The Lie algebras given in Tables 1 and 2 are the candidates for being quasi-exactly solvable Lie algebras, i.e. we might expect them to admit $\mathcal{N}$ a finite dimensional module of smooth functions $\mathcal{N}$. In the investigation for these explicit finite dimensional modules, some new restrictions are imposed on the 1-cocycles $F$. Indeed, as for the quasi-exactly solvable Lie algebras in lower dimensions, it comes out that a finite dimensional module exists only if the values of the functions $\eta_{v}$ are taken in a certain discrete set. For this reason, this restriction is named quantization condition. The quasi-exactly solvable Lie algebras and their fixed modules can be found in Tables 3 and 4 for, respectively, the type I and the selected type III Lie algebras. The first column use the same classification numbers as in Tables 1 and 2 and a representative for the non-trivial quantized 1-cocycles is exhibited in the second column. Finally, $\mathcal{N}$, the finite dimensional $\mathfrak{g}$-modules of functions are described in the last column. Once again the detailed calculations are repetitive and the essence of the work can be grasped with one or two examples, together with the following general principles.

1. A finite dimensional module for the trivial Lie algebra $\mathfrak{g}=\{p\}$ is defined as an $x$-translation module. For instance, any space spanned by a finite set of functions of the form

$$
h=\sum_{i=0}^{n} g^{i}(y, z) x^{i},
$$


along with all their $x$ derivatives, is an $x$-translation module. This particular case of $x$-translation module is referred as a semi-polynomial $x$-translation module. The most general $x$-translation module is obtained by a direct sum

$$
\mathcal{N}=\bigoplus_{\lambda \in \Lambda} \mathcal{N}_{\lambda}, \quad \mathcal{N}_{\lambda}=\widehat{\mathcal{N}_{\lambda}} e^{\lambda x}
$$

where $\widehat{\mathcal{N}_{\lambda}}$ are semi-polynomial $x$-translation modules and the exponents are taken in a finite set $\Lambda$, read [9] for more details. Obviously, the $y$, and the $z$-translation modules are defined the exact same way.

2. If the Lie algebra $\mathfrak{g}$ under consideration contains the two differential operators $p$ and $x p$, the module $\mathcal{N}$ will be an $x$-translation module and the operator $x p$ will impose extra constraints. Firstly, all the exponents $\lambda$ need be zero. Otherwise, for an non-zero exponent $\lambda$, the degree in $x$ of the generating functions in the module $\widehat{\mathcal{N}_{\lambda}}$ would be unbounded, contradicting the finite dimensionality of $\mathcal{N}$. Moreover, if $h=\sum_{i=0}^{n} g^{i}(y, z) x^{i}$ belongs to the module $\mathcal{N}$, the function $x h_{x}$ also needs to belong to that module. Note that both functions have the same degree in $x$ and are linearly independent if $h$ is not a monomial. Thus, by an appropriate linear combination of these two functions, one can reduce the number of summands in $h$. By iterating this process, each generating function can be reduced to a monomial in $x$. Thus, $h=g(y, z) x^{i}$ where $g(y, z)$ belongs to $G^{i}$ a finite set of functions in $y$ and $z$. Since $\mathcal{N}$ is a $x$-translation module, $h_{x}=i g(y, z) x^{i-1}$ is also a function in $\mathcal{N}$, hence $g(y, z)$ needs to be also contained in $G^{i-1}$. Therefore, the module $\mathcal{N}$ decomposes into the following direct sum

$$
\mathcal{N}=\bigoplus x^{i} g_{k}^{i}(y, z), \quad i=0, \ldots, n, \quad k=0, \ldots, l_{i}
$$

where all the functions $g_{k}^{i}(y, z)$ belong to $G^{i}$ a finite set and where $G^{i} \subseteq G^{i-1}$.

3. Likewise, if a Lie algebra $\mathfrak{g}$ contains the elements $p, q, x p$, and $y q$, a general finite dimensional $\mathfrak{g}$-module for this Lie algebra will be at most

$$
\mathcal{N}=\bigoplus x^{i} y^{j} g_{k}^{i, j}(z), \quad i=0, \ldots, n, \quad j=0, \ldots, m, \quad k=0, \ldots, l_{(i, j)},
$$

where the functions $g_{k}^{i, j}(z)$ belong to $G^{(i, j)}$, a finite set of functions of $z$ satisfying $G^{(i, j)} \subseteq$ $G^{(i-1, j)} \cap G^{(i, j-1)}$.

A simple method to describe these modules is to represent each generating function $x^{i} y^{j} g^{i, j}(z)$ by a point $(i, j)$, in the Cartesian plane. If a vertex $(i, j)$ belongs to the diagram, since $\mathcal{N}$ is an $x y$-translation module, the vertices $(i-1, j)$ and $(i, j-1)$ must also sit in the diagram. To complete the description, a finite set $G^{(i, j)}$ is associated to each of these vertices, with the same restriction as above. For instance, such module $\mathcal{N}$ can be represented by

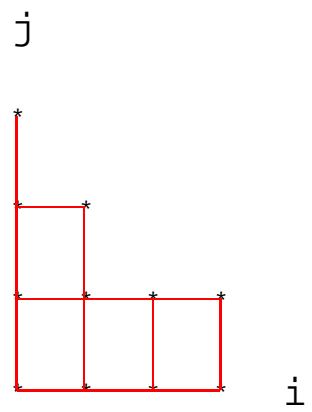


with all the sets $G^{(i, j)}$ being equal to $\left\{z, e^{z}\right\}$, with the exception of $G^{(3,1)}$ that contains only the function $z$. It is then straightforward to verify that this module is indeed

$$
\begin{aligned}
& \mathcal{N}=\operatorname{Span}\left\{0, z, e^{z}, x z, x e^{z}, y z, y e^{z}, x^{2} z, x^{2} e^{z}, x y z, x y e^{z}, y^{2} z,\right. \\
&\left.y^{2} e^{z}, x^{3} z, x^{3} e^{z}, x^{2} y z, x^{2} y e^{z}, x y^{2} z, x y^{2} e^{z}, y^{3} z, y^{3} e^{z}, x^{3} y z\right\},
\end{aligned}
$$

and that it is a $\mathfrak{g}$-module for the Lie algebra $\mathfrak{g}=\{p, x p, y, y q\}$.

4. If the Lie algebra $\mathfrak{g}$ contains the differential operators $p, q, x p, y q$ and $y p$, from the three previous principles, the generators for a $\mathfrak{g}$-module are given by $h=x^{i} y^{j} g^{i, j}(z)$. After applying the operator yp on $h$, the resulting function reads as $i x^{i-1} y^{j+1} g^{i, j}(z)$. Thus, iterating this operator, we conclude that all the functions $x^{i-r} y^{j+r} g^{i, j}(z)$ must belong to $\mathcal{N}$, for $r \leq i$. Since $p$ and $q$ also belong to the algebra, all the functions $x^{a} y^{b} g^{i, j}(y, z)$ with $a \leq i, b \leq j$ and $a+b \leq c$ must belong to $\mathcal{N}$. This condition can be expressed by the following inclusion $G^{(i, j)} \subseteq G^{(i-1, j)} \cap G^{(i, j-1)} \cap G^{(i-1, j+1)}$, and observe that the first set $G^{(i-1, j)}$ can be omitted without affecting the condition. To summarize, the $\mathfrak{g}$-module will be at most

$$
\mathcal{N}=\bigoplus x^{i} y^{j} g_{k}^{i, j}(z), \quad i=0, \ldots, n, \quad j=0, \ldots, m, \quad k=0, \ldots, l_{(i, j)},
$$

where the functions $g_{k}^{i, j}(z)$ belong to $G^{(i, j)}$, a finite set of functions with $G^{(i, j)} \subseteq G^{(i-1, j+1)} \cap$ $G^{(i, j-1)}$.

Once again it is possible to represent such module by a diagram along with a set of functions $G^{(i, j)}$ for each vertex of the diagram. The restrictions for these sets are $G^{(i, j)} \subseteq$ $G^{(i-1, j+1)} \cap G^{(i, j-1)}$ and the conditions on the vertices are slightly different from the one in the previous example. Indeed, if a vertex $(i, j)$, belongs to the diagram, the two vertices $(i-1, j+1)$ and $(i, j-1)$ must also belong to the diagram. Note again that this implies that the vertex $(i-1, j)$ also lies in the diagram. For instance the diagram,

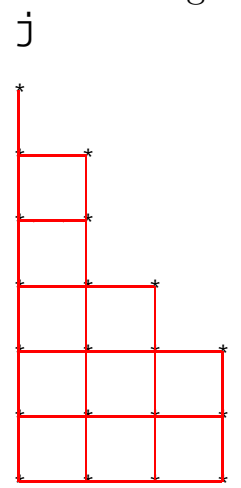

i

together with twenty appropriate sets of functions $G^{(i, j)}$ for each vertex, would generate a $\mathfrak{g}$-module for the algebra $\mathfrak{g}=\{p, q, x p, y q, y p\}$.

5. Finally, if the elements $p, q, x p, y q, y p$ and $x q$ sit in the Lie algebra under consideration, the module will be at most

$$
\mathcal{N}=\bigoplus x^{i} y^{j} g_{k}^{i, j}(z), \quad i+j=0, \ldots, n, \quad k=0, \ldots, l_{i, j}
$$

where the functions $g_{k}^{i, j}(z)$ belong to $G^{(i+j)}$, a finite set of functions with $G^{(l)} \subseteq G^{(l-1)}$. Indeed, consider $h=x^{i} y^{j} g^{i, j}(z)$, a generator of bi-degree $i+j=c$. Since $x q[h]$ and $y p[h]$ must also lie in $\mathcal{N}$, all the functions $x^{a} y^{b} g^{i, j}(z)$ with $a+b=c$ will belong to $\mathcal{N}$. Hence $g^{(i, j)} \in G^{(a, b)}$ and, reciprocally, $g^{(a, b)} \in G^{(i, j)}$. Thus for all pairs $(a, b)$ with $a+b=c$, 
the finite sets $G^{(a, b)}$ are identical and it is therefore well defined to pose $G^{(a, b)}=G^{(a+b)}$. Obviously, since $\mathcal{N}$ is a $x y$-translation module, the following inclusions hold $G^{(l)} \subseteq G^{(l-1)}$.

For these modules, the possible diagrams are more restricted and have necessarily the shape of a staircase. Also, instead of assigning one set of functions to each vertex, such a set is coupled to all the vertices having same total degree $i+j$. For instance, the module represented by the diagram

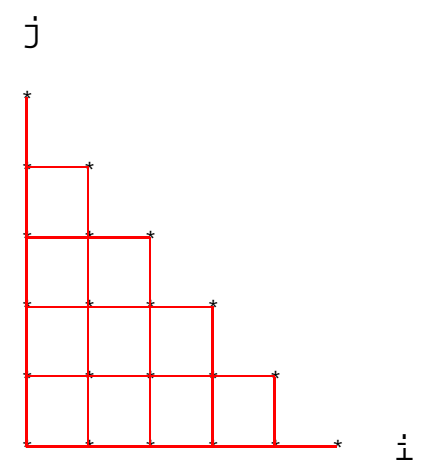

will be completely determined after fixing six sets of function in $z$. Note that this choice must respect the inclusion $G^{(l)}(z) \subseteq G^{(l-1)}(z)$, for $i=1, \ldots, 5$.

Table 1. Cohomology for the type I Lie algebras of vector fields, $m=\{1\}$.

\begin{tabular}{|lll|}
\hline & Generators & Cocycles \\
\hline \hline 1 & $\left\{p, q, x p, y q, x q, y p, x^{2} p+x y q, x y p+y^{2} q\right\}$ & $x p+K(z), x^{2} p+x y q+x K(z)$, \\
& & $y q+K(z), x y p+y^{2} q+y K(z)$ \\
\hline 2 & $\{x q, x p-y q, y p$, & 0 \\
& $\left.Z^{1}(z) p, \ldots, Z^{l}(z) p, Z^{1}(z) q, \ldots, Z^{l}(z) q\right\}$ & \\
\hline 3 & $\{x q, x p-y q, y p, x p+y q$, & $x p+y q+K(z)$ \\
& $\left.Z^{1}(z) p, \ldots, Z^{l}(z) p, Z^{1}(z) q, \ldots, Z^{l}(z) q\right\}$ & \\
\hline 4 & $\left\{p, q, x p, y q, x q, y p, x^{2} p+x y q, x y p+y^{2} q, r\right\}$ & $x^{2} p+x y q+c x, x y p+y^{2} q+c y$ \\
\hline 5 & $\left\{x q, x p-y q, y p, z^{k} e^{\lambda_{l} z} p, z^{k} e^{\lambda_{l} z} q, r\right\}$, & 0 \\
& $k \leq n_{l}, l=0, \ldots, b$ \\
\hline 6 & $\{x q, x p-y q, y p, x p+y q$, \\
& $\left.z^{k} e^{\lambda_{l} z} p, z^{k} e^{\lambda_{l} z} q, r\right\}, k \leq n_{l}, l=0, \ldots, b$ & \\
\hline 7 & $\left\{p, q, x p, y q, x q, y p, x^{2} p+x y, q, x y p+y^{2} q, r, z r\right\}$ & $x^{2} p+x y q+c x, x y p+y^{2} q+c y$ \\
\hline 8 & $\left\{x q, x p-y q, y p, p, z p, \ldots, z^{l} p\right.$, & 0 \\
& $\left.q, z q, \ldots, z^{l} q, r, z r+a(x p+y q)\right\}$ & \\
\hline 9 & $\left\{x q, x p-y q, y p, x p+y q, p, z p, \ldots, z^{l} p\right.$, & 0 \\
& $\left.q, z q, \ldots, z^{l} q, r, z r\right\}, k \leq n_{l}, l=0, \ldots, b$ & \\
\hline 10 & $\{p, q, x p, y q, x q, y p$, & $x^{2} p+x y q+c x, x y p+y^{2} q+c y$, \\
& $\left.x^{2} p+x y q, x y p+y^{2} q, r, z r, z^{2} r\right\}$ & $z^{2} r+d z$ \\
\hline 11 & $\left\{x q, x p-y q, y p, p, z p, \ldots, z^{l} p, q, z q, \ldots, z^{l} q\right.$, \\
& $\left.r, z r+\frac{a}{2}(x p+y q), z^{2} r+a z(x p+y q)\right\}$ & $z^{2} r+a z(x p+y q)+c z$ \\
\hline 12 & $\left\{x q, x p-y q, y p, x p+y q, p, z p, \ldots, z^{l} p\right.$, & $z^{2} r+a z(x p+y q)+c z$ \\
& $\left.q, z q, \ldots, z^{l} q, r, z r, z^{2} r+a z(x p+y q)\right\}$ & \\
\hline \hline
\end{tabular}

Note here that $b, l$ and $n_{l}$ are positive integers, $a, c, d, k$ and $\lambda_{l}$ are arbitrary constants and $Z^{1}(z), \ldots, Z^{l}(z)$ and $K(z)$ functions of $z$. 
Table 2. Cohomology for some type III Lie algebras of vector fields, $m=\{1\}$.

\begin{tabular}{|c|c|c|}
\hline & Generators & Cocycles \\
\hline $4 \mathrm{~A}$ & $\begin{array}{l}\left\{p, y q, q, x q, \ldots, x^{t} q+r, \ldots, x^{i} q+\left(\begin{array}{l}t \\
i\end{array}\right) x^{t-i} r, \ldots\right. \\
\left.x^{s} q+\left(\begin{array}{l}t \\
s\end{array}\right) x^{s-t} r, x p-t z r, y q+z r\right\}, 0 \leq t \leq s\end{array}$ & 0 \\
\hline $4 \mathrm{C}$ & $\begin{array}{l}\left\{q, x q, \ldots, x^{s} q, p, y q, x p, x^{l} y^{n-b} r, z r\right\} \\
0 \leq b \leq n, l \leq l_{0}+s b\end{array}$ & 0 \\
\hline $4 \mathrm{D}$ & $\left\{q, x q, \ldots, x^{s} q, p, y q, x p, r, z r, z^{2} r\right\}$ & $z^{2} r+c z$ \\
\hline $5 \mathrm{~A}^{*}$ & $\begin{array}{l}\left\{q+r, x q+x r, \ldots, x^{s} q+x^{s} r, p\right. \\
\left.x p, y q+z r, x^{2} p+s x y q+s x z r\right\}\end{array}$ & $x^{2} p+s x y q+s r+c x$ \\
\hline $5 \mathrm{C}$ & $\begin{array}{l}\left\{q, x q, \ldots, x^{s} q, p, y q, x p, x^{2} p+s x y q+\left(l_{0}+s \tilde{n}\right) x z r\right. \\
\left.x^{l} y^{\tilde{n}-b} r, z r\right\}, 0 \leq b \leq \tilde{n}, l \leq l_{0}+s b\end{array}$ & $x^{2} p+s x y q+\left(l_{0}+s \tilde{n}\right) r+c x$ \\
\hline $5 \mathrm{D}$ & $\left\{q, x q, \ldots, x^{s} q, p, y q, x p, x^{2}+s x y q, r, z r, z^{2} r\right\}$ & $x^{2} p+s x y q+c x, z^{2} r+d z$ \\
\hline $7 \mathrm{C}$ & $\left\{p, 2 x p+y q, x^{2} p+x y q, x^{l} y^{-n} r, z r\right\}, 0 \leq l \leq n$ & $x^{2} p+x y q+c y^{2}$ \\
\hline $17 \mathrm{~A}_{1}$ & $\left\{p, q, x p+z r, y q, x^{2} p+(2 x+a z) z r, y^{2} q\right\}$ & $\begin{array}{l}x^{2} p+(2 x+a z) z r+b x+c z \\
y^{2} q+d y\end{array}$ \\
\hline $17 \mathrm{~A}_{2}$ & $\left\{p, q, x p+a z r, y q+z r, x^{2} p+2 a x z r, y^{2} q+2 y z r\right\}$ & $\begin{array}{l}x^{2} p+2 a x z r+c x \\
y^{2} q+2 y z r+d y\end{array}$ \\
\hline $17 \mathrm{C}$ & $\begin{array}{l}\left\{p, q, x p, y q, x^{2} p+l_{0} x z r, y^{2} q+p_{0} y z r, x^{l} y^{p} r\right\} \\
l \leq l_{0}, b \leq b_{0}\end{array}$ & $\begin{array}{l}x^{2} p+l_{0} x z r+c x \\
y^{2} q+b_{0} y z r+d y\end{array}$ \\
\hline $17 \mathrm{D}$ & $\left\{p, x p, x^{2} p, q, y p, y^{2} q, r, z r, z^{2} r\right\}$ & $x^{2} p+a x, y^{2} q+c y, z^{2} r+d z$ \\
\hline
\end{tabular}

Note here that $b, b_{0}, l, l_{0}, \tilde{n}, s$ and $t$ are positive integers, $a, c$ and $d$ are arbitrary constants. Remark that Amaldi's Lie algebra $5 \mathrm{~A}$ is not a Lie algebra. Indeed, for the space spanned by $\left\{q, x q, \ldots, x^{t} q+\right.$ $\left.r, \ldots, x^{t+i} q+\left(\begin{array}{c}t+i \\ t\end{array}\right) x^{i} r, \ldots, x^{s} q+\left(\begin{array}{c}s \\ t\end{array}\right) x^{s-t} r, p, y p, x p-t z r, y q+z r, x^{2} p+s x y q+(s-2 t) x z r, x^{l} y^{n-b} r, z r\right\}$ to be a Lie algebra, the parameter $t$ needs to be zero. We then get the Lie algebra $5 \mathrm{~A}^{*}$ given in the table.

This set of principles is of great help in the determination of the possible $\mathfrak{g}$-modules $\mathcal{N}$ for each Lie algebras of first order differential operators $\mathfrak{g}$ described in Tables 1 and 2. Depending on the elements contained in the Lie algebra studied, we started our search of $\mathfrak{g}$-module based on the general module given in this guideline. Once again, the computations are tedious and it would not be relevant to detail each of them. We will concentrate on the same Lie algebras as in the previous step of this classification problem, that is the type I Lie algebras cases 1 and 10.

Type I, case 1. Since the Lie algebra contains the differential operators $p, q, x p$, and $y p$, from the principle (3), the most general module $\mathcal{N}$ will be spanned by functions of the form $h=$ $x^{i} y^{j} g^{i, j}(z)$, where $g^{i, j}(z)$ belongs to $G^{(i, j)}$. We now consider the operator $T=x^{2} p+x y q+3 x K(z)$ in the algebra $\mathfrak{g}$ and its action on $h=x^{n} y^{a} g^{n, a}(z)$ a generator of $\mathcal{N}$ with maximal exponent in $x$. Thus

$$
\begin{aligned}
T[h] & =n x^{n+1} y^{a} g^{n, a}(z)+a x^{n+1} y^{a} g^{n, a}(z)+3 K(z) x^{n+1} y^{a} g^{n, a}(z) \\
& =(n+a+3 K(z)) x^{n+1} y^{a} g^{n, a}(z) .
\end{aligned}
$$

Since the exponent in $x$ was taken to be maximal, this imposes that $K(z)$ is indeed a constant $K$ equal to $-\frac{n+a}{3}$. Symmetrically, by considering $Q=x y p+y^{2} q+3 y K(z)$ and $h=x^{b} y^{m} g^{b, m}(z)$, a function with maximal exponent in $y$, the following equality holds $K=-\frac{b+m}{3}$. For this to be possible, we necessarily have $n+a=b+m$. Consequently, the differential operators $x q$ and $y p$ belong to the Lie algebra $\mathfrak{g}$ and the module $\mathcal{N}$ is given by the principle (5). Also note that the operators $x p$ and $y q$ force both $a$ and $b$ to be zero. Otherwise $x^{n+1} y^{a-1} g^{n, a}(z)$ and $x^{b-1} y^{m+1} g^{b, m}(z)$ would be in $\mathcal{N}$, contradicting the maximality of $n$ and $m$. Thus $3 K(z)=-n$ 
Table 3. Type I quasi-exactly solvable Lie algebras of differential operators and their fixed modules.

\begin{tabular}{|lll|}
\hline & Quantization condition & Fixed module \\
\hline \hline 1 & $x^{2} p+x y q-n x, x y p+y^{2} q-n y$ & $\begin{array}{l}\left\{x^{i} y^{j} g(z) \mid i+j \leq n, g(z) \in G^{(i+j)}\right\}, \\
\text { for } G^{(l)} \text { finite with } G^{(l)} \subseteq G^{(l-1)}\end{array}$ \\
\hline 2 & 0 & $\begin{array}{l}\left\{x^{i} y^{j} g(z) \mid i+j \leq n, g(z) \in G^{(i+j)}\right\}, \\
\text { for } G^{(l)} \text { finite with } Z^{i} G^{(l)} \subseteq G^{(l-1)}\end{array}$ \\
& & $\left\{x^{i} y^{j} g(z) \mid i+j \leq n, g(z) \in G^{(i+j)}\right\}$, \\
& for $G^{(l)}$ finite, and $Z^{i} G^{(l)} \subseteq Z^{i} G^{(l-1)}$ \\
& 0 & $\left\{x^{i} y^{j} z^{k} e^{\lambda_{l} z} \mid i+j \leq n, k \leq m_{l}, l=0, \ldots, p\right\}$ \\
\hline 4 & $x^{2} p+x y q-n x, x y p+y^{2} q-n y$ & $\left\{x^{i} y^{j} z^{k} e^{\lambda_{l} z} \mid i+j \leq n, z^{k} e^{\lambda_{l} z} \in G^{(i+j)}\right\}$, \\
\hline 5 & 0 & for $G^{(l)}$ a finite $z$-translation module, \\
& & and $Z^{i} G^{(l)} \subseteq G^{(l-1)}$ for $Z^{i}=z^{k} e^{\lambda_{l} z} p$ \\
& & $\left\{x^{i} y^{j} z^{k} e^{\lambda_{l} z} \mid i+j \leq n, k \leq m m_{l}, l=0, \ldots, p\right\}$, \\
& for $G^{(l)}$ a finite $z$-translation module,, \\
6 & 0 & and $Z^{i} G^{(l)} \subseteq G^{(l-1)}$ for $Z^{i}=z^{k} e^{\lambda_{l} z} p$ \\
& & $\left\{x^{i} y^{j} z^{k} \mid i+j \leq n, k \leq m\right\}$ \\
\hline 7 & $x^{2} p+x y q-n x, x y p+y^{2} q-n y$ & $\left\{x^{i} y^{j} z^{k} \mid i+j \leq n, l(i+j)+k \leq m\right\}$ \\
\hline 8 & 0 & $\left\{x^{i} y^{j} z^{k} \mid i+j \leq n, l(i+j)+k \leq m\right\}$ \\
\hline 9 & 0 & $\left\{x^{i} y^{j} z^{k} \mid i+j \leq n, k \leq m\right\}$ \\
\hline 10 & $x^{2} p+x y q-n x, x y p+y^{2} q-n y$ & \\
\hline$z^{2} r-m z$ & $\left\{x^{i} y^{j} z^{k} \mid i+j \leq n, a(i+j)+k \leq m\right\}$ \\
\hline 11 & $z^{2} r+a z(x p+y q)-m z$ & $\left\{x^{i} y^{j} z^{k} \mid i+j \leq n, a(i+j)+k \leq m\right\}$ \\
\hline 12 & $z^{2} r+a z(x p+y q)-m z$ & \\
\hline \hline
\end{tabular}

Note here that $m$ and $n$ are positive integers.

Table 4. Some of the type III quasi-exactly solvable Lie algebras of differential operators and their fixed modules.

\begin{tabular}{|c|c|c|}
\hline & Quantization condition & Fixed module \\
\hline $4 \mathrm{~A}$ & 0 & $\left\{x^{i} y^{j} z^{k} \mid i+s j+(s-t) k \leq n, j \leq m_{y}, k \leq m_{z}\right\}$ \\
\hline $4 \mathrm{C}$ & 0 & $\begin{array}{l}\left\{x^{i} y^{j} z^{k} \mid i+s j+\left(l_{0}+s n\right) k \leq n, k \leq b, j \leq b_{k}\right\} \\
\text { with } b_{k-1} \geq b_{k}+n\end{array}$ \\
\hline $4 \mathrm{D}$ & $z^{2} r-m z$ & $\left\{x^{i} y^{j} z^{k} \mid i+s j \leq n, k \leq m\right\}$ \\
\hline $5 \mathrm{~A}^{*}$ & $x^{2} p+s x y q+s r-n x$ & $\left\{x^{i} y^{j} z^{k} \mid i+s(j+k) \leq n, j \leq m_{y}, k \leq m_{z}\right\}$ \\
\hline $5 \mathrm{C}$ & $x^{2} p+s x y q+\left(l_{0}+s \tilde{n}\right) x z r-n x$ & $\begin{array}{l}\left\{x^{i} y^{j} z^{k} \mid i+s j+\left(l_{0}+s \tilde{n}\right) k \leq n, k \leq b, j \leq b_{k}\right\} \\
\text { with } b_{k-1} \geq b_{k}+\tilde{n}\end{array}$ \\
\hline $5 \mathrm{D}$ & $x^{2} p+s x y q-n x, z^{2}-m z$ & $\left\{x^{i} y^{j} z^{k} \mid i+s j \leq n, k \leq m\right\}$ \\
\hline $7 \mathrm{C}$ & 0 & 0 \\
\hline $17 \mathrm{~A}_{1}$ & $x^{2} p+(2 x+a z) z r-m_{x} x, y^{2} q-m_{y} y$ & $\left\{x^{i} y^{j} \mid i \leq m_{x}, j \leq m_{y}\right\}$ \\
\hline $17 \mathrm{~A}_{2}$ & $x^{2} p+2 a x z r-m_{x} x, y^{2} q+2 y z r-m_{y} y$ & $\left\{x^{i} y^{j} z^{k} \mid i+2 a k \leq m_{x}, j+2 k \leq m_{y}, k \leq m_{z}\right\}$ \\
\hline $17 \mathrm{C}$ & $x^{2} p+l_{0} x z r-m_{x} x, y^{2} q+b_{0} y z r-m_{y} y$ & $\left\{x^{i} y^{j} z^{k} \mid i+l_{0} k \leq m_{x}, j+b_{0} k \leq m_{y}, k \leq m_{z}\right\}$ \\
\hline $17 \mathrm{D}$ & $x^{2} p-m_{x} x, y^{2} q-m_{y} y, z^{2} r-m_{z} z$ & $\left\{x^{i} y^{j} z^{k} \mid i \leq m_{x}, j \leq m_{y}, k \leq m_{z}\right\}$ \\
\hline
\end{tabular}

Note here that $m, n, m_{x}, m_{y}$ and $m_{z}$ are positive integers. 
and the module

$$
\mathcal{N}=\left\{x^{i} y^{j} g^{i, j}(z) \mid i+j \leq n, g^{i, j}(z) \in G^{(i+j)}\right\}, \quad \text { where } \quad G^{(l)} \subseteq G^{(l-1)}
$$

is fixed by all the differential operators in $\mathfrak{g}$. Therefore it is possible conclude that the Lie algebra

$$
\mathfrak{g}=\operatorname{Span}\left\{p, q, x p, y q, x q, y p, x^{2} p+x y q-n x, x y p+y^{2} q-n y, 1\right\},
$$

is quasi-exactly solvable with respect to the finite dimensional $\mathfrak{g}$-module $\mathcal{N}$.

Type I, case 10. Since the case 10 Lie algebra contains the case 1 Lie algebra, its module $\mathcal{N}$ will be at most the module given in (8). Observe first that the constant $c$ in the case 10 Lie algebra has to be the negative integer $-n$. Furthermore, the operator $r$ imposes $\mathcal{N}$ to be a $z$ translation module and the operator $z r$ forces $G^{(l)}$ to be generated by monomials. Then, for $z^{m}$ a monomial of maximal degree in $G^{(l)}$, the function $h=x^{i} y^{l-i} z^{m}$ belongs to $\mathcal{N}$. Since $z^{2} r+d z$ belongs to the Lie algebra,

$$
z^{2} r+d z[h]=m x^{i} y^{l-i} z^{m+1}+d x^{i} y^{l-i} z^{m+1}=[m+d] x^{i} y^{l-i} z^{m+1},
$$

should belong to the $\mathfrak{g}$-module $\mathcal{N}$. Thus, from the maximality of the degree in $z$, the constant $d$ has to be the negative integer $-m$. Since the argument must hold for every set $G^{(l)}$, they will all share the same monomial of maximal degree $m$. We can therefore conclude that the Lie algebra

$$
\mathfrak{g}=\operatorname{Span}\left\{p, q, x p, y q, x q, y p, x^{2} p+x y q-n x, x y p+y^{2} q-n y, r, z r, z^{2} r-m z, 1\right\},
$$

is quasi-exactly solvable with respect to the module

$$
\mathcal{N}=\left\{x^{i} y^{j} z^{k} \mid i+j \leq n, k \leq m\right\} .
$$

To summarize, a partial classification of quasi-exactly solvable Lie algebras of first order differential operators was accomplished in this section and the description of these Lie algebras $\mathfrak{g}$, along with their $\mathfrak{g}$-modules, can be found in Tables 1-4. In principle, it would be possible to achieve a complete classification using similar arguments. However this gigantic work would require a colossal amount of time. Nevertheless this partial classification is a good starting point for seeking new quasi-exactly solvable Schrödinger operators in three dimensions. In that scope, the next section is devoted to the description of few new quasi-exactly solvable Schrödinger operators.

\section{New quasi-exactly solvable Schrödinger operators in three dimensions}

Recall that in the general classification problem, once the quasi-exactly solvable Lie algebras of differential operators $\mathfrak{g}$ are determined, the next step is to construct second order differential operators $\mathcal{H}$ that are locally equivalent to Schrödinger operators. Given $\mathfrak{g}$, one of the Lie algebras of first order differential operators obtained in the previous section, we obtain a second order differential operator $\mathcal{H}$ by letting

$$
\mathcal{H}=\sum_{a, b=1}^{m} C_{a b} T^{a} T^{b}+\sum_{a=1}^{m} C_{a} T^{a}+C_{0}, \quad \text { where } \quad T^{a} \in \mathfrak{g},
$$

as illustrated previously. Then, one has to choose the coefficients $C_{a b}, C_{a}, C_{0}$ in such a way that the closure conditions $d \omega=0$ are satisfied. Given $T^{a}=\xi^{a i} \partial_{i}+\eta^{a}$, the closure conditions 
are the Frobenius compatibility conditions for the overdetermined system

$$
\sum_{a, b=1}^{m} \xi^{a i}\left[C_{a b}\left(\sum_{j=1}^{n}\left(\xi^{b j} \frac{\partial \alpha}{\partial x^{j}}+\frac{\partial \xi^{b j}}{\partial x^{j}}\right)-2 \eta^{b}\right)-C_{a}\right]=0
$$

where $\alpha=\lambda+\frac{1}{2} \ln (g)$ and $\mu=e^{\lambda}$ is the gauge factor. Finally, we need to bear in mind that the last step in the classification is to verify that the operators are normalizable, i.e. the functions in $\tilde{\mathcal{N}}$, the module obtained after the gauge transformation, need to be square integrable. These operators will therefore have the property that part of their spectrum can be explicitly computed.

We have now in hand a large variety of generating quasi-exactly solvable Lie algebras $\mathfrak{g}$. The door is therefore wide open to the construction of numerous new quasi-exactly solvable Schrödinger operators in three dimensions. However the Schrödinger operators described in this paper are built only from two of these new quasi-exactly solvable Lie algebras: the type III cases $17 \mathrm{D}$ and case $5 \mathrm{~A}^{*}$. The reader can therefore see that many more examples can be constructed using this method together with the results of the previous section.

\subsection{Type III, case $17 \mathrm{D},(\mathfrak{s l}(2) \times \mathfrak{s l}(2) \times \mathfrak{s l}(2))$}

The first two families of normalizable quasi-exactly solvable Schrödinger operators displayed in this section are similar to the operator given in the example (1). However, these two examples are more general. Indeed, for these two operators, the type III case 17D quasi-exactly solvable Lie algebra $\mathfrak{g}$ is spanned by the first order differential operators

$$
p, \quad x p, \quad x^{2} p-m_{x} x, \quad q, \quad y q, \quad y^{2} q-m_{y} y, \quad r, \quad z r, \quad z^{2} r-m_{z} z
$$

where $m_{x}, m_{y}$ and $m_{z}$ are non negative integers and the module $\mathcal{N}_{m_{x} m_{y} m_{z}}$ is generated by the $\left(m_{x}+1\right)\left(m_{y}+1\right)\left(m_{z}+1\right)$ monomials

$$
x^{i} y^{j} z^{k} \quad \text { where } \quad 0 \leq i \leq m_{x}, \quad j \leq m_{y} \quad \text { and } \quad k \leq m_{z} .
$$

\subsubsection{First example}

The first family of operators is constructed with the following choice of coefficients

$$
\begin{aligned}
C_{a b} & =\left(\begin{array}{ccccccccc}
A & 0 & 1 & 0 & 0 & 0 & 0 & 0 & 1 \\
0 & B & 0 & 0 & 0 & 0 & 0 & 1 & 0 \\
1 & 0 & C & 0 & 0 & 0 & 1 & 0 & 0 \\
0 & 0 & 0 & 2 A & 0 & 0 & 0 & 0 & 0 \\
0 & 0 & 0 & 0 & 2 B & 0 & 0 & 0 & 0 \\
0 & 0 & 0 & 0 & 0 & 2 C & 0 & 0 & 0 \\
0 & 0 & 1 & 0 & 0 & 0 & A & 0 & 1 \\
0 & 1 & 0 & 0 & 0 & 0 & 0 & B & 0 \\
1 & 0 & 0 & 0 & 0 & 0 & 1 & 0 & C
\end{array}\right), \\
C_{c} & =\left[0,0,0,-2 A m_{x},-2 B m_{y},-2 C m_{z}, 0,0,0\right] \text { and } C_{0}=-A m_{x}-B m_{y}-C m_{z} .
\end{aligned}
$$

From the order two terms of these second order differential operators, the induced contravariant metric $g^{(i j)}$ is obtained and reads as

$$
\left(\begin{array}{ccc}
A\left(x^{2}+1\right)^{2} & 0 & \left(x^{2}+1\right)\left(z^{2}+1\right) \\
0 & B y^{4}+2(B+1) y^{2}+B & 0 \\
\left(x^{2}+1\right)\left(z^{2}+1\right) & 0 & C\left(z^{2}+1\right)
\end{array}\right) .
$$


The determinant of the matrix is $\widetilde{g}=(1-A C)\left(x^{2}+1\right)^{2}\left(B y^{4}+2(B+1) y^{2}+B\right)\left(z^{2}+1\right)^{2}$ and one easily verifies, for $A, B$ and $C$ positive and $A B>1$, that the matrix is positive definite on $\mathbb{R}^{3}$. The operator can therefore be written as

$$
-2 \mathcal{H}=\Delta+\vec{V}+U \text {, }
$$

where $\Delta$ is the Laplace-Beltrami operator related to the metric (10) and where

$$
\vec{V}=-2\left(x^{2}+1\right)\left(A m_{x} x+m_{z} z\right) p-2 m_{y}\left(B y^{3}+B y+y\right) q-2\left(z^{2}+1\right)\left(C m_{z} z+m_{x} x\right) r .
$$

From a direct computation, the closure conditions are verified and the gauge factor required to gauge transform $\mathcal{H}$ into a Schrödinger operator $\mathcal{H}_{0}$ is given by

$$
\mu=\left(x^{2}+1\right)^{\frac{-m_{x}}{2}}\left(B y^{4}+2(B+1) y^{2}+B\right)^{\frac{-m_{y}}{4}}\left(z^{2}+1\right)^{\frac{-m_{z}}{2}} .
$$

Once the transformation is performed, the equivalent operator reads as

$$
-2 \mathcal{H}_{0}=\Delta+U
$$

where the potential of the Schrödinger operator $-\frac{U}{2}$ is given by a rational function in $y$.

Note that the same three factors arise in both $\mu$ and $\widetilde{g}$. This will simplify our computations while testing the square integrability of the functions in $\widetilde{\mathcal{N}}$. Indeed, a function in $\widetilde{\mathcal{N}}$ is given by $h=\mu x^{i} y^{j} z^{k}$ where the exponents $i, j$ and $k$ are smaller or equal to $m_{x}, m_{y}$ and $m_{z}$ respectively. Our aim is to show that the triple integral

$$
\iiint_{\mathbb{R}^{3}}\left(\mu x^{i} y^{j} z^{k}\right)^{2} \sqrt{g} d x d y d z
$$

is finite. Obviously, it is sufficient to show the convergence of this integral for the monomials of maximal exponent. We can therefore focus on

$$
\iiint_{\mathbb{R}^{3}} \frac{x^{2 m_{x}} y^{2 m_{y}} z^{2 m_{z}}}{\left(x^{2}+1\right)^{m_{x}+1}\left(B y^{4}+2(B+1) y^{2}+B\right)^{\frac{m_{y}}{2}+\frac{1}{2}}\left(z^{2}+1\right)^{m_{z}+1}} d x d y d z .
$$

Using Fubini's theorem, this triple integral can be factored into the product of three integrals

$$
\begin{aligned}
& \int_{-\infty}^{\infty} \frac{x^{2 m_{x}}}{\left(x^{2}+1\right)^{m_{x}+1}} d x, \quad \int_{-\infty}^{\infty} \frac{y^{2 m_{y}}}{\left(B y^{4}+2(B+1) y^{2}+B\right)^{\frac{m_{y}}{2}+\frac{1}{2}}} d y, \quad \text { and } \\
& \int_{-\infty}^{\infty} \frac{z^{2 m_{z}}}{\left(z^{2}+1\right)^{m_{z}+1}} d z,
\end{aligned}
$$

each of which is easily shown to be convergent. We therefore have in hand a normalizable quasi-exactly solvable Schrödinger operator and it is feasible to determine explicitly part of its spectrum.

For instance, if we fix $m_{x}=0, m_{y}=2$ and $m_{z}=1$, few manipulations lead to the six eigenfunctions of the operator $\mathcal{H}$ restricted to $\mathcal{N}$. Indeed, with this choice of parameters, the $\mathfrak{g}$-module is

$$
\mathcal{N}=\left\{1, y, y^{2}, z, y z, y^{2} z\right\},
$$

and the transformation matrix to be diagonalized reads as

$$
\left(\begin{array}{ccrccc}
-2 & 0 & 2 & B & 0 & 0 \\
0 & -4-2 B & 0 & 0 & 0 & 0 \\
2 B & 0 & -2 & 0 & 0 & 0 \\
0 & 0 & 0 & -2 & 0 & 2 B \\
0 & 0 & 0 & 0 & -4-2 B & 0 \\
0 & 0 & 0 & 2 B & 0 & -2
\end{array}\right) .
$$


Once the diagonalization is performed, three different eigenvalues $\lambda_{1}=-4-2 B, \lambda_{2}=-2-2 B$, and $\lambda_{3}=-2+2 B$ are obtained, each of them having multiplicity two. The six eigenfunctions are respectively

$$
\begin{aligned}
& \psi_{1,1}=y, \quad \psi_{1,2}=y z, \quad \psi_{2,1}=1+y^{2}, \quad \psi_{2,2}=-1+y^{2}, \\
& \psi_{3,1}=-z+y^{2} z, \quad \psi_{3,2}=z+y^{2} z .
\end{aligned}
$$

Consequently, we obtain three multiplicity two eigenvalues of the Schrödinger operator $\mathcal{H}_{0}$ : $\widetilde{\lambda_{1}}=1-B, \widehat{\lambda_{2}}=1+B$ and $\widetilde{\lambda_{3}}=2+B$, and the six scaled eigenfunctions are

$$
\begin{array}{ll}
\widetilde{\psi_{1,1}} & =\mu y, \quad \widetilde{\psi_{1,2}}=\mu y z, \quad \widetilde{\psi_{2,1}}=\mu\left(1+y^{2}\right), \quad \widetilde{\psi_{2,2}}=\mu\left(-1+y^{2}\right), \\
\widetilde{\psi_{3,1}} & =\mu\left(-z+y^{2} z\right), \quad \widetilde{\psi_{3,2}}=\mu\left(z+y^{2} z\right) .
\end{array}
$$

As mentioned previously, the metric (10) is positive definite on $\mathbb{R}^{3}$, hence Riemannian, and one can compute that the Riemann curvature tensor is zero everywhere. The change of variables that leads to a Cartesian coordinate system is given by

$$
X=\arctan x, \quad Y=\int \frac{1}{\sqrt{B y^{4}+2(B+1) y^{2}+B}}, \quad Z=\arctan z,
$$

where we have some flexibility on $B$ to adjust the roots of the elliptic integral. Note that this operator is a good illustration of the modified Turbiner's conjecture in three dimensions proved in [6]. Indeed, the generating Lie algebra $\mathfrak{g}$ is imprimitive and the leaves of the foliation are surfaces, the Riemann curvature tensor is zero and, as expected, the potential is separable since it depends on only one variable.

\subsubsection{Second example}

With the same representation of Lie algebra by first order differential operators but a different choice of coefficients, one constructs another family of second order differential operators $\mathcal{H}$. Indeed, with

$$
\begin{aligned}
C_{a b} & =\left(\begin{array}{ccccccccc}
A & 0 & 1 & 0 & 0 & 0 & 0 & 0 & \lambda \\
0 & D & 0 & 0 & 0 & 0 & 0 & \beta & 0 \\
1 & 0 & B & 0 & 0 & 0 & 1 & 0 & 0 \\
0 & 0 & 0 & 2 A & 0 & 0 & 0 & 0 & 0 \\
0 & 0 & 0 & 0 & \beta D+C & 0 & 0 & 0 & 0 \\
0 & 0 & 0 & 0 & 0 & 2 \lambda B & 0 & 0 & 0 \\
0 & 0 & 1 & 0 & 0 & 0 & A & 0 & \lambda \\
0 & \beta & 0 & 0 & 0 & 0 & 0 & \beta C & 0 \\
\lambda & 0 & 0 & 0 & 0 & 0 & \lambda & 0 & \lambda^{2} B
\end{array}\right), \\
C_{c} & =\left[0,0,0,-2 A m_{x},-\beta D\left(1+2 m_{y}\right)+C,-2 \lambda B m_{z}, 0,0,0\right] \\
C_{0} & =-A m_{x}-B m_{y}-C m_{z},
\end{aligned}
$$

a family of operators $\mathcal{H}$ is obtained and one easily verifies that all these operators are equivalent to Schrödinger operators $\mathcal{H}_{0}$. Note that this family of operators is slightly more general than the family obtained in the first example. However, some of the details are lengthy and are omitted for brevity sake. The induced contravariant metric $g^{(i j)}$ is given by:

$$
\left(\begin{array}{ccc}
A\left(x^{2}+1\right)^{2} & 0 & \left(x^{2}+1\right)\left(\lambda z^{2}+1\right) \\
0 & \beta C y^{4}+y^{2}(2 \beta+\beta D+C)+D & 0 \\
\left(x^{2}+1\right)\left(\lambda z^{2}+1\right) & 0 & \left.B\left(\lambda z^{2}+z\right)\right)
\end{array}\right),
$$


and it is positive definite on $\mathbb{R}^{3}$ provided $A, B, C, D$ and $\beta$ are positive and $A B>1$. Its determinant is $\widetilde{g}=(A B-1)\left(x^{2}+1\right)^{2}\left(\beta C y^{4}+2 \beta y^{2}+\beta D y^{2}+y^{2} C+D\right)\left(\lambda z^{2}+1\right)^{2}$, and the gauge factor required is the product of three functions in $x, y$ and $z$ respectively. After the gauge transformation the new potential is again a rational function involving only the $y$ variable and the Riemann curvature tensor is null again. The following change of variables leads to Cartesian coordinates

$$
\begin{aligned}
& X=\arctan x, \quad Y=\int \frac{1}{\sqrt{\beta C y^{4}+2 \beta y^{2}+2 \beta D y^{2}+y^{2} c+D}} d y, \\
& Z=\frac{\arctan \sqrt{\lambda} z}{\sqrt{\lambda}},
\end{aligned}
$$

where we have some flexibility on $\beta, C$ and $D$ to adjust the roots of the elliptic integral $\mathrm{Y}$.

However, we do not know if these operators are all normalizable. But, if we fix $C=\beta D$, the gauge transformation simplifies and becomes, once again, very similar to the determinant of the metric (11). Indeed

$$
\mu=\left(x^{2}+1\right)^{-\frac{m_{x}}{2}}\left(\beta^{2} D y^{4}+2 \beta y^{2}(1+D)+D\right)^{-\frac{m_{y}}{4}}\left(\lambda z^{2}+1\right)^{-\frac{m_{z}}{2}}
$$

and one verifies, the exact same way as in the previous example, that the functions in $\widetilde{\mathcal{N}}$ are square integrable. Therefore, for any choice of integers $m_{x}, m_{y}$, and $m_{z}$, one would obtain $\left(m_{x}+1\right)\left(m_{y}+1\right)\left(m_{z}+1\right)$ eigenfunctions in the spectrum of the Schrödinger operator $\mathcal{H}_{0}$.

For instance, if we fix $\lambda=1, \beta=5$ and the three parameters $m_{x}, m_{y}$ and $m_{z}$ to be 1 , one gets two eigenvalues, -3 and -7 of multiplicity four, and the following eight eigenfunctions

$$
\begin{array}{llrl}
\widetilde{\psi_{-7,1}}=\mu(-1+x z), & \widetilde{\psi_{-7,2}}=\mu(y-x y z), \\
\widetilde{\psi_{-7,3}}=\mu(x y+y z), & \widetilde{\psi_{-7,4}}=\mu(x+z), \\
\widetilde{\psi_{-3,1}}=\mu(y+x y z), & \widetilde{\psi_{-3,2}}=\mu(-x+z), \\
\widetilde{\psi_{-3,3}}=\mu(-x y+y z), & \widetilde{\psi_{-3,4}}=\mu(1+x z) .
\end{array}
$$

Note that the nodal surfaces can described easily in this coordinate system. Indeed, since $\mu$ is always positive, the nodal surfaces are simply the zero loci of polynomials. For these eight eigenfunctions, the surfaces are given by the zeros of degree two factorizable polynomials and one easily gets the following pictures.

$x+z$

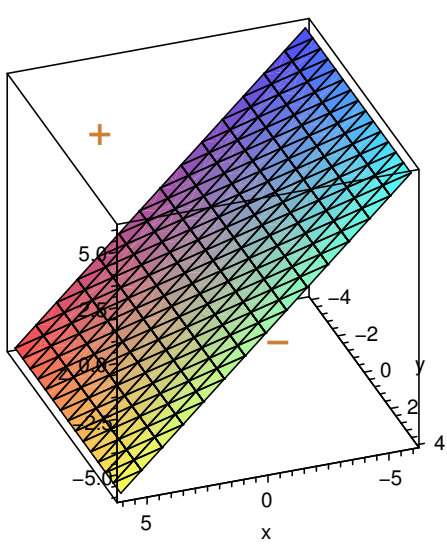

$-x+z$

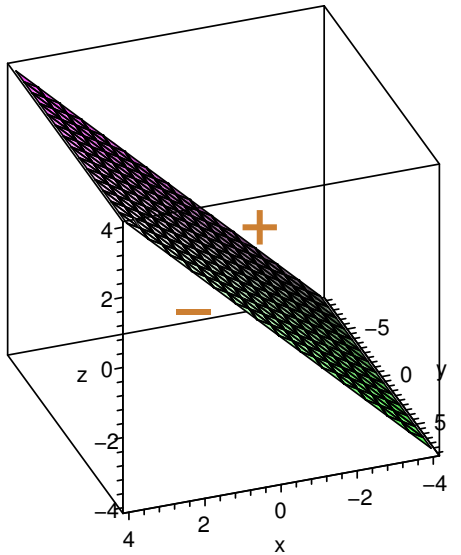



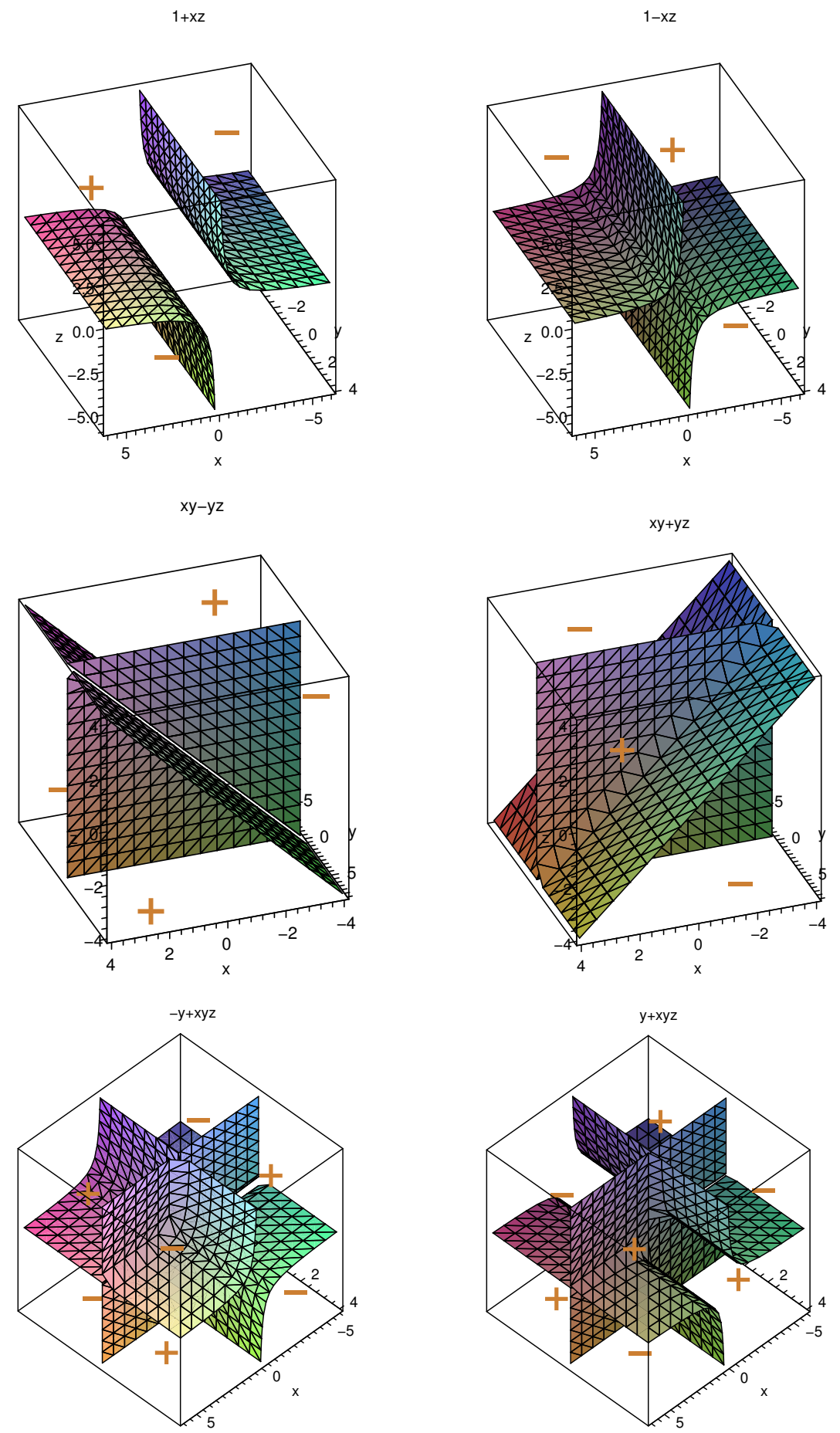

\subsection{Type III, case $5 \mathrm{~A}^{*}, \mathfrak{s l}(2) \ltimes \mathbb{C}^{s+1}$}

For the last example, we consider the type III case $5 \mathrm{~A}^{*}$ quasi-exactly solvable Lie algebra and we fix the parameter $s$ to be one. This Lie algebra $\mathfrak{g}$ is therefore spanned by the following six first order differential operators

$$
p, \quad q+r, x q+x r, \quad x p, \quad y q+z r, \quad \text { and } \quad x^{2} p+x y q+x z r-n x,
$$

and from the Table 4 , the $\mathfrak{g}$-module of function is given by

$$
\mathcal{N}=\left\{x^{i} y^{j} z^{k} \mid i+j+k \leq n, j \leq m_{y}, k \leq m_{z}\right\}
$$


where $n, m_{y}$ and $m_{z}$ are non-negative integers. A family of Schrödinger operators on $\mathbb{R}^{3} \backslash\{x=y\}$ is obtained from the following choice of coefficients,

$$
\begin{aligned}
C_{a b} & =\left(\begin{array}{cccccc}
A & 0 & 0 & 0 & 0 & 0 \\
0 & B & 0 & 0 & 0 & 0 \\
0 & 0 & C & 0 & 0 & 0 \\
0 & 0 & 0 & 0 & 0 & 0 \\
0 & 0 & 0 & 0 & D & 0 \\
0 & 0 & 0 & 0 & 0 & 0
\end{array}\right), \\
C_{c} & =[0,0, C, 0,-2(1+m) D, 0], \quad \text { and } C_{0}=(1+m)^{2} D,
\end{aligned}
$$

where the parameters $A, B, C$, and $D$ are positive. The induced contravariant metric is given by

$$
g^{(i j)}=\left(\begin{array}{ccc}
C x^{2}+A & 0 & 0 \\
0 & D y^{2}+B & D y z+B \\
0 & D y z+B & D z^{2}+B
\end{array}\right)
$$

its determinant is $\widetilde{g}=B D\left(C x^{2}+A\right)(y-z)^{2}$ and the metric is positive definite on $\mathbb{R}^{3} \backslash\{x=y\}$. Before performing the gauge transformation the operator reads as

$$
\begin{aligned}
-2 \mathcal{H}= & \Delta+(2 C x-C m x) p+(-D y-2 D m y) q+(-D z-2 D m z) r \\
& -1 / 2 C m+1 / 4 C m^{2}+(1+m)^{2} D,
\end{aligned}
$$

and one easily verifies that the operator respects the closure condition. The gauge factor required to obtain a Schrödinger operator is

$$
\mu=\left(C x^{2}+A\right)^{\frac{1-m}{4}}(y-z)^{\frac{-2 m-3}{2}},
$$

and once again, contains the same factors as the determinant of the covariant metric. Finally, after the gauge transformation, the Schrödinger operator reads as,

$$
-2 \mathcal{H}_{0}=\Delta+U
$$

where $U$ depends on the three variables. Although, it is not known if the functions in $\widetilde{\mathcal{N}}$ are square integrable on the domain $\mathbb{R}^{3} \backslash\{x=y\}$.

Note that for this example, the scalar curvature is constant and depends on the parameter $D$ while the Riemann curvature tensor is equal to

$$
\frac{-1}{B(y-z)^{2}} d y d z d y d z .
$$

However, the potential does not seem to be separable.

\section{Acknowledgements}

The research is supported by a NSERC Grant \#RGPIN 105490 - 2004 and a McGill Graduate Studies Fellowship. I would like to thank Niky Kamran, for all the encouragement and the precious advice he gave me. 


\section{References}

[1] Amaldi U., Contributo alla determinazione dei gruppi continui finiti dello spazio ordinario, part I, Giornale di matematiche di Battaglini per il progresso degle studi nelle universita italiane 39 (1901), 273-316. Amaldi U., Contributo alla determinazione dei gruppi continui finiti dello spazio ordinario, part II, Giornale di matematiche di Battaglini per il progresso degle studi nelle universita italiane 40 (1902), 105-141.

[2] Barut A.O., Böhm A., Dynamical groups and mass formula, Phys. Rev. (2) 139 (1965), B1107-B1112.

[3] Böhm A., Ne'eman Y., Barut A.O. (Editors), Dynamical groups and spectrum generating algebras, World Scientific, Singapore, 1988.

[4] Böhm A., Ne'eman Y., Dynamical groups and spectrum generating algebras, in Dynamical Groups and Spectrum Generating Algebras, World Scientific, Singapore, 1988, 3-68.

[5] Dothan Y., Gell-Mann M., Ne'eman Y., Series of hadron energy levels as representation of non-compact groups, Phys. Lett. 17 (1965), 148-151.

[6] Fortin-Boisvert M., Turbiner's conjecture in three dimensions, J. Geom. Phys., to appear, math.DG/0612621.

[7] Fulton W., Harris J., Representation theory, Springer-Verlag, 1991.

[8] González-López A., Hurturbise J., Kamran N., Olver P.J., Quantification de la cohomologie des algebres de Lie de champs de vecteurs et fibres en droites sur des surfaces complexes compactes, C. R. Acad. Sci. Paris Sér. I Math. 316 (1993), 1307-1312.

[9] González-López A., Kamran N., Olver P.J., Lie algebras of first order differential operators in two complex variables, Canadian Math. Soc. Conf. Proc., Vol. 12, Amer. Math. Soc., Providence, R.I., 1991, 51-84.

[10] González-López A., Kamran N., Olver P.J., Normalizability of one-dimensional quasi-exactly solvable Schrödinger operators, Comm. Math. Phys. 153 (1993), 118-146.

[11] González-López A., Kamran N., Olver P.J., New quasi-exactly solvable Hamiltonians in two dimensions, Comm. Math. Phys. 159 (1994), 503-537.

[12] González-López A., Kamran N., Olver P.J., Quasi-exactly solvable Lie algebras of first order differential operators in two complex variables, J. Phys. A: Math. Gen. 24 (1991), 3995-4008.

[13] González-López A., Kamran N., Olver P.J., Quasi-exact solvablility, Contemp. Math. 160 (1994), $113-139$.

[14] González-López A., Kamran N., Olver P.J., Real Lie algebras of differential operatord and quasi-exacly solvable potentials, Philos. Trans. Roy. Soc. London Ser. A 354 (1996), 1165-1193.

[15] Goshen S., Lipkin H.J., A simple independent particle system having collective properties, Ann. Phys. 6 (1959), 301-309.

[16] Gruber B., Otsuka T. (Editors), Symmetries in science VII: spectrum-generating algebras and dynamic symmetries in physics, Plenum Press, New York, 1993.

[17] Iachello F., Levine R.D., Algebraic theory of molecules, Oxford Univ. Press, Oxford, (1995).

[18] Lie S., Theorie der Transformationsgruppen, Vol. III, Chelsea Publishing Company, New York, 1970.

[19] Milson R., Multi-dimensional Lie algebraic operators, Ph.D. Thesis, McGill University, 1995.

[20] Milson R., Representation of finite-dimensional Lie algebras by first order differential operators. Some local results in the transitive case, J. London Math. Soc. (2) 52 (1995), 285-302.

[21] Miller W., Jr., Lie theory and special functions, Academic Press, New York, 1968.

[22] Milson R., Richter D., Quantization of cohomology in semi-simple Lie algebras, J. Lie Theory 8 (1998), 401-414.

[23] Shifman M.A., Turbiner A.V., Quantal problems with partial algebraization of the spectrum, Comm. Math. Phys. 126 (1989), 347-365.

[24] Turbiner A.V., Quasi-exactly solvable problems and $\mathfrak{s l}(2)$ algebras, Comm. Math. Phys. 118 (1988), $467-474$.

[25] Ushveridze A.G., Quasi-exactly solvable models in quantum mechanics, Soviet J. Particles and Nuclei 20 (1989), 504-528.

[26] Ushveridze A.G., Quasi-exactly solvable models in quantum mechanics, IOP Publ., Bristol, 1994. 